\title{
Histone H3 Threonine 11 Phosphorylation Is Catalyzed Directly by the Meiosis-Specific Kinase Mek1 and Provides a Molecular Readout of Mek1 Activity in Vivo
}

\author{
Ryan Kniewel, ${ }^{*,+, 1,2}$ Hajime Murakami, ${ }^{*, 2}$ Yan Liu, ${ }^{\neq, 3}$ Masaru Ito, ${ }^{\S, 4}$ Kunihiro Ohta, ${ }^{\S}$ Nancy M. Hollingsworth, ${ }^{\ddagger}$ \\ and Scott Keeney,t,**,5 \\ *Molecular Biology Program, Memorial Sloan Kettering Cancer Center, New York, New York 10065, 'Weill Cornell Graduate \\ School of Medical Sciences, New York, New York 10065, ₹Department of Biochemistry and Cell Biology, Stony Brook University, \\ New York 11794, §Department of Life Sciences, The University of Tokyo, 153-8902, Japan, and **Howard Hughes Medical \\ Institute, Memorial Sloan Kettering Cancer Center, New York, New York 10065 \\ ORCID IDs: 0000-0003-3462-6450 (R.K.); 0000-0002-7022-6887 (H.M.); 0000-0003-3300-6444 (N.M.H.); 0000-0002-1283-6417 (S.K.)
}

\begin{abstract}
Saccharomyces cerevisiae Mek1 is a CHK2/Rad53-family kinase that regulates meiotic recombination and progression upon its activation in response to DNA double-strand breaks (DSBs). The full catalog of direct Mek1 phosphorylation targets remains unknown. Here, we show that phosphorylation of histone H3 on threonine 11 (H3 T11ph) is induced by meiotic DSBs in S. cerevisiae and Schizosaccharomyces pombe. Molecular genetic experiments in S. cerevisiae confirmed that Mek1 is required for H3 T11ph and revealed that phosphorylation is rapidly reversed when Mek 1 kinase is no longer active. Reconstituting histone phosphorylation in vitro with recombinant proteins demonstrated that Mek1 directly catalyzes H3 T11 phosphorylation. Mutating H3 T11 to nonphosphorylatable residues conferred no detectable defects in otherwise unperturbed meiosis, although the mutations modestly reduced spore viability in certain strains where Rad51 is used for strand exchange in place of Dmc1. H3 T11 ph is therefore mostly dispensable for Mek1 function. However, H3 T11ph provides an excellent marker of ongoing Mek1 kinase activity in vivo. Anti-H3 T11ph chromatin immunoprecipitation followed by deep sequencing demonstrated that $\mathrm{H} 3 \mathrm{~T} 11$ ph was highly enriched at presumed sites of attachment of chromatin to chromosome axes, gave a more modest signal along chromatin loops, and was present at still lower levels immediately adjacent to DSB hotspots. These localization patterns closely tracked the distribution of Red1 and Hop1, axis proteins required for Mek1 activation. These findings provide insight into the spatial disposition of Mek1 kinase activity and the higher order organization of recombining meiotic chromosomes.
\end{abstract}

KEYWORDS Meiosis; Histone phosphorylation; Mek1; DNA damage response; Chromosome structure

EIOTIC recombination initiates with DNA doublestrand breaks (DSBs) made by the topoisomerase-like transesterase Spo11 (Lam and Keeney 2014). DSBs occur

Copyright (C) 2017 by the Genetics Society of America

doi: https://doi.org/10.1534/genetics.117.300359

Manuscript received June 6, 2017; accepted for publication October 5, 2017; published Early Online October 5, 2017

Supplemental material is available online at www.genetics.org/lookup/suppl/doi:10. 1534/genetics.117.300359/-/DC1.

${ }^{1}$ Present address: Department of Environmental Biology, Centro de Investigaciones Biológicas, Consejo Superior de Investigaciones Científicas, 28040 Madrid, Spain. ${ }^{2}$ These authors contributed equally to this work.

${ }^{3}$ Present address: Northwell Health Systems, 1983 Marcus Ave., New Hyde Park, New York, NY 11040.

${ }^{4}$ Present address: Department of Microbiology and Molecular Genetics, University of California, Davis, CA 95616.

${ }^{5}$ Corresponding author: Howard Hughes Medical Institute, Memorial Sloan Kettering Cancer Center, 1275 York Ave., Box 97, New York, NY 10065. E-mail: s-keeney@ski.mskcc.org throughout the genome, often but not always in hotspots that in Saccharomyces cerevisiae mostly overlap with nucleosomedepleted gene promoters (Ohta et al. 1994; Wu and Lichten 1994; Baudat and Nicolas 1997; Pan et al. 2011). Repair of meiotic DSBs by recombination helps form physical connections between homologous chromosomes that allow the chromosomes to segregate accurately at the first meiotic division (MI) (Hunter 2015). Because recombination defects can lead to mutations and/or aneuploidy, meiotic DSB repair is highly regulated (Subramanian and Hochwagen 2014; Hunter 2015).

A critical component of this regulation in yeast is Mre4/ Mek1, a meiosis-specific paralog of the Rad53 checkpoint effector kinase (Rockmill and Roeder 1991; Leem and Ogawa 1992). In response to Spo11-generated DSBs, the kinases Tel1 and/or Mec1 (orthologs of mammalian ATM and ATR, 
respectively) become activated and phosphorylate the chromosome axis-associated protein Hop1 among other substrates (Carballo et al. 2008; Cheng et al. 2013; Penedos et al. 2015). The Forkhead-associated (FHA) domain of Mek1 then binds phosphorylated Hop1, resulting in Mek1 recruitment to chromosome axes where Mek1 undergoes activation (involving trans-autophosphorylation on T327 in its activation loop) and stabilizes Hop1 phosphorylation via positive feedback (Niu et al. 2005, 2007; Carballo et al. 2008; Chuang et al. 2012; Penedos et al. 2015). Activated Mek1 promotes interhomolog bias in recombination, that is, the preferential use of a homologous chromosome rather than sister chromatid as the template for DSB repair (Niu et al. 2005; Carballo et al. 2008; Goldfarb and Lichten 2010; Kim et al. 2010; Hong et al. 2013; Lao et al. 2013; Subramanian et al. 2016). Mek1 does so in part by phosphorylating the Rad54 protein on threonine 132 (T132) (Niu et al. 2007, 2009). Rad54 is a member of the Swi2/Snf2 DNA-dependent, ATPase chromatin remodeling family and is a binding partner of the strand exchange protein Rad51 (Heyer et al. 2006). Mek1-dependent phosphorylation of Rad54 attenuates the interaction with Rad51, allowing the meiosis-specific strand exchange protein Dmc1 to predominate (Niu et al. 2009). Mek1 also directly phosphorylates the T40 residue of Hed1; this stabilizes the Hed1 protein and thereby promotes its function as a negative regulator of Rad51 strand exchange activity (Callender et al. 2016). Additionally, Mek1 promotes the repair of interhomolog strand invasion intermediates through a pathway required for chromosome synapsis and the generation of crossovers, whose distribution shows interference (Chen et al. 2015). Finally, MEK1 is required for checkpoint arrest or delay of meiotic progression in response to unrepaired DSBs (Lydall et al. 1996; Xu et al. 1997).

The full array of direct Mek1 phosphorylation substrates remains unknown, as only three direct targets have been definitively proven thus far: Mek1 itself, Rad54, and Hed1 (Niu et al. 2007, 2009; Callender et al. 2016). Additional Mek1-dependent phospho-proteins have been identified by mass spectrometry and other approaches, including T11 of histone H3 (Govin et al. 2010; Suhandynata et al. 2016). However, a number of Mek1-dependent phosphorylation events are known or suspected to be indirect (Suhandynata et al. 2016). For example, Mek1 is required for phosphorylation of the synaptonemal complex protein Zip1, but the kinase directly responsible is Cdc7-Dbf4, not Mek1 (Chen et al. 2015). Moreover, H3 T11 phosphorylation has been reported as being catalyzed in vegetative cells by other kinases [the pyruvate kinases Pyk1 and, to a lesser extent, Pyk2 (Li et al. 2015)], which could in principle be regulated by Mek1 in meiosis. Therefore, whether H3 T11 is a direct substrate for Mek1 remains to be established.

Mek1 activity plays out in the context of elaborate higher order chromosome structures. Early in meiotic prophase, sister chromatids form cooriented arrays of DNA loops that are anchored along a linear proteinaceous axis (Zickler and Kleckner 1999; Kleckner 2006). Prominent components of these axes include sister chromatid cohesion proteins (including the meiosis-specific Rec8 subunit), Mek1, Hop1, and another meiosis-specific chromosome structural protein, Red1 (Smith and Roeder 1997; Bailis and Roeder 1998; Klein et al. 1999; Panizza et al. 2011).

In cytological experiments, immunostaining foci of recombination proteins are axis associated, indicating that recombination occurs in proximity to axes (reviewed in Zickler and Kleckner 2015). However, there is an anticorrelation between the DNA sequences preferentially bound by axis proteins (Rec8, Hop1, and Red1) and the DNA sequences that often experience Spo11-induced DSBs, which suggests that recombination usually involves the DNA in chromatin loops rather than the DNA embedded in axes (Gerton et al. 2000; Blat et al. 2002; Pan et al. 2011; Panizza et al. 2011). To reconcile this paradox, the "tethered-loop/axis complex" (TLAC) model proposes that DNA segments residing on chromatin loops incur DSBs but are recruited, or tethered, to axes by interactions between recombination proteins and axis proteins (Kleckner 2006; Panizza et al. 2011). The TLAC model provides a framework for understanding spatial organization of recombining chromosomes, but there is as yet little direct molecular data demonstrating the proposed functional interactions between axes and DSB sites.

How Mek1 fits into this proposed organization also remains unknown. Immunocytology suggests that Mek1 protein is localized primarily on axes (Bailis and Roeder 1998; Subramanian et al. 2016), supported by the dependence of Mek1 activity on axis proteins (Niu et al. 2007; Carballo et al. 2008). However, Mek1 exerts its known recombination-controlling activity (directly or indirectly) at sites of DSBs. The TLAC model can account for Mek1 acting at both places, but where Mek1 kinase activity actually occurs remains unexplored because of a lack of a molecular marker for the active kinase.

In this study, we demonstrate that Mek1 directly phosphorylates histone H3 T11 in response to meiotic DSBs in S. cerevisiae. Phosphorylated H3 T11 (H3 T11ph) is dispensable for Mek1 function during unperturbed meiosis, so the purpose of this phosphorylation remains unclear. Nevertheless, we demonstrate the utility of $\mathrm{H} 3 \mathrm{~T} 11 \mathrm{ph}$ as a direct molecular marker for active Mek1 by examining the genomewide localization of $\mathrm{H} 3 \mathrm{~T} 11 \mathrm{ph}$. Our findings suggest that Mek1 exerts its activity at axis association sites but also across chromatin loops.

\section{Materials and Methods}

\section{Strains and histone mutagenesis strategy}

S. cerevisiae and Schizosaccharomyces pombe strains are listed in Supplemental Material, Table S1. S. pombe strains were generously provided by G. Smith, Fred Hutchinson Cancer Research Center. Histone gene deletion strains and plasmids expressing H3 T11 mutants from Govin et al. (2010) were generously provided by S. Berger, University of Pennsylvania. 
S. cerevisiae strains were of the SK1 strain background. Because of concerns about effects of plasmid (in)stability on the ability to score phenotypes of histone mutants and to reliably measure meiotic parameters because of cell-to-cell heterogeneity within a culture (see Results), we opted to avoid plasmid shuffle systems that have been used by others (Ahn et al. 2005; Govin et al. 2010). Instead, strategies involving stable integration or gene replacement were employed, as follows.

Histone gene replacements: S. cerevisiae histone genes are arranged in divergently oriented pairs expressing either $\mathrm{H} 3$ and $\mathrm{H} 4$ or $\mathrm{H} 2 \mathrm{~A}$ and $\mathrm{H} 2 \mathrm{~B}$; there are two of each pair, i.e., two copies encoding each histone. The S10A and T11V mutations were introduced into plasmid-borne copies of HHT1 and HHT2 by QuikChange site-directed mutagenesis (Agilent Technologies). These mutant alleles were then introduced sequentially into SK1 strain SKY165 by one-step gene replacements using DNA fragments containing $\geq 270 \mathrm{bp}$ arms of homology. Targeting constructs included selectable drug resistance markers: kanMX4 $\sim 366$ bp downstream of the HHT1 ORF and hphMX4 250 bp downstream of HHT2.

Stable integration of histone gene cassettes: A histone cassette integration strategy was employed using pRS305based plasmids (Sikorski and Hieter 1989) integrated into the leu2::hisG locus. Integrations were performed to try to maintain balanced gene dosage for the four core histones. The parental strain for the $\mathrm{H} 2 \mathrm{~A} / \mathrm{H} 2 \mathrm{~B} / \mathrm{H} 3 / \mathrm{H} 4$ histone cassette integrations was created in a multistep process by first transforming a pRS316-based URA3 histone cassette-covering plasmid containing a single copy of each histone gene (pRK12; HTA1-HTB1, HHT2-HHF2) into diploid SKY165. Next, the histone gene pairs, HHT2-HHF2 and HTA1-HTB1 [which are required for proper meiosis (Norris and Osley 1987)], were deleted sequentially and replaced with the hphMX and natMX markers, respectively. The deletions were confirmed by Southern blot and the strain was sporulated to yield a $\mathrm{Ura}^{+}, \mathrm{Nat}^{\mathrm{R}}, \mathrm{Hyg}^{\mathrm{R}}, M A T \alpha$ haploid. A second MATa haploid strain was created by sequentially deleting the other (nonessential) histone gene pairs, HTA2-HTB2 and HHT1-HHF1, which were replaced by the kanMX and natMX markers, respectively, and confirmed by Southern blot. These two haploids were mated to form a compound heterozygote, tetrads were dissected, and then resulting haploids carrying all four histone gene-pair deletions were mated to form a histone integration host strain (SKY2283) with the genotype: hht1-hhf1s::kanMX/", hht2-hhf2s::natMX/", hta1-htb1s::hphMX/", hta2-htb2S::natMX/", pRK12[CEN/ARS, URA3, HTA1-HTB1, HHF2HHT2].

A parental strain for the $\mathrm{H} 3 / \mathrm{H} 4$ histone cassette integrations was created by dissecting tetrads from the hht2-hhf2A:: natMX/", $p R K 12$ strain described above prior to deletion of HTA1-HTB1. This dissection yielded a $\mathrm{Ura}^{+}, \mathrm{Nat}^{\mathrm{R}}$, MATa haploid that was crossed with the second haploid strain described above (hta2-htb2s::natMX, hht1-hhf1s::kanMX). Tetrad dissection yielded MATa and MAT $\alpha$ haploid progeny
(SKY3166 and SKY3167, respectively) with the following genotype: hht1-hhf1s::kanMX, hhf2-hht2A::natMX, hta2htb2A::natMX, pRK12.

All histone mutant integration constructs were created by QuikChange site-directed mutagenesis. The first was an H3/ H4 replacement using a pRS305-based plasmid (pRK77) containing LEU2, HHT2-HHF2 that was linearized by AflII digestion to target integration to leu2::hisG and transformed into haploids SKY3166 and SKY3167. The second was an $\mathrm{H} 2 \mathrm{~A} / \mathrm{H} 2 \mathrm{~B} / \mathrm{H} 3 / \mathrm{H} 4$ replacement using a pRS305-based plasmid (pRK24) containing LEU2, HTA1-HTB1 and HHF2HHT2 that was linearized by AflII digestion and transformed into diploid SKY2283. In both cases, the core histonecovering plasmid pRK12 was counterselected by growth on 5-fluoroorotic acid (5-FOA). Colony PCR of $\mathrm{Leu}^{+}, \mathrm{Ura}^{-}$ transformants was used to verify the proper integration into the leu2::hisG locus using primer sets flanking both junctions as well as verification of the mutations in $h t a 1$ and $h h t 2$ by engineered restriction enzyme site polymorphisms and/or sequencing. In the case of the SKY3166/3167 transformants, haploid integrants were subsequently mated to create diploids. SKY2283 hemizygous integrants were sporulated to produce haploid progeny that were then mated to create homozygous diploids.

\section{S. cerevisiae and S. pombe cultures}

S. cerevisiae was cultured at $30^{\circ}$ with asynchronous vegetative (cycling) cultures in YPD (1\% yeast extract, $2 \%$ peptone, $2 \%$ dextrose). Camptothecin treatment $(20 \mu \mathrm{M})$ was performed for $2 \mathrm{hr}$ at $30^{\circ}$ in $250 \mathrm{ml}$ flasks shaking at $250 \mathrm{rpm}$ in $10 \mathrm{ml}$ cultures of SKY165 at an initial cell density of $\sim 9 \times$ $10^{7} \mathrm{cell} \mathrm{s} / \mathrm{ml}$. An untreated culture was incubated in parallel, while a separate $10 \mathrm{ml}$ aliquot in a vented T-75 flask was exposed to $\mathrm{X}$ rays for $60 \mathrm{~min}$ at room temperature using an X-RAD 225C X-ray irradiator (Precision X-Ray) corresponding to a dose of $400 \mathrm{~Gy}$. Alternatively, $10 \mathrm{ml}$ of culture at $\sim 7 \times$ $10^{7}$ cells $/ \mathrm{ml}$ was exposed to $\mathrm{X}$ rays for $60 \mathrm{~min}$ on ice, with untreated cells also held on ice. With both exposure conditions, cells were subsequently allowed to recover at $30^{\circ}$, shaking at $225 \mathrm{rpm}$ for $60 \mathrm{~min}$ (room temperature exposure) or 30 min (exposure on ice) before fixing in $20 \%$ trichloroacetic acid (TCA), pelleting, and storage at $-80^{\circ}$ until extract preparation.

For inhibition of Mek1-as in vivo, an SKY3095 culture was divided equally $4 \mathrm{hr}$ after transfer to sporulation medium and $10 \mu \mathrm{l} 100 \%$ DMSO was added to half while the other half received a $1 \mu \mathrm{M}$ final concentration of 1-NA-PP1 [1-(1,1-dimethylethyl)-3(1-naphthalenyl)-1 $H$-pyrazolo[3,4-d]pyrimidin-4-amine] dissolved in DMSO (Wan et al. 2004). The return-to-growth recombination assays using arg4 heteroalleles were carried out in triplicate as described (Martini et al. 2006). Pulsedfield gel electrophoresis and Southern blotting on DNA from meiotic cultures prepared using the standard presporulation medium (SPS) method was performed as described (Murakami et al. 2009). Plasmid shuffling and meiotic cultures using plasmids and the SK1 histone gene deletion 
strain, obtained from S. Berger, were carried out as described (Govin et al. 2010).

S. pombe haploid pat1-114 sporulation was carried out as described (Hyppa and Smith 2009). For S. cerevisiae meiotic cultures, strains were thawed on YPG plates (1\% yeast extract, $2 \%$ peptone, $3 \%$ glycerol, $2 \%$ agar) and incubated for $\sim 2$ days, streaked for single colonies on YPD plates, and grown for $\sim 2$ days. Single diploid colonies were inoculated in $5 \mathrm{ml}$ YPD and grown overnight. Cultures were diluted in YPA (1\% yeast extract, $2 \%$ peptone, and $1 \%$ potassium acetate) to $\sim 1.2 \times 10^{6}$ cells $/ \mathrm{ml}$ and grown for $13.5 \mathrm{hr}$ at $225 \mathrm{rpm}$ for chromatin immunoprecipitation (ChIP), and $250 \mathrm{rpm}$ for all other experiments. Cells were pelleted, washed in sterile water, and resuspended in the same preculture volume of $2 \%$ potassium acetate to a density of $\sim 2-3 \times$ $10^{7}$ cells $/ \mathrm{ml}$. This corresponds to $0 \mathrm{hr}$ of the meiotic time course. Sporulation was at $225 \mathrm{rpm}$ for ChIP and $250 \mathrm{rpm}$ for all other experiments. Unless indicated otherwise, statistical significance of spore viabilities was assessed by Fisher's exact test, treating dissected tetrads as a random spore population. Meiotic progression was assessed in culture aliquots fixed with $50 \%$ ethanol and stained with $5 \mu \mathrm{g} / \mathrm{ml} \mathrm{4',6-}$ diamidino-2-phenylindole (DAPI).

\section{Whole-cell extracts and western blotting}

Culture aliquots with an $\mathrm{OD}_{600}$ of 10 for $S$. pombe or $\sim 3.2 \times$ $10^{8}$ cells for S. cerevisiae were washed in $20 \%$ TCA, pelleted, and stored at $-80^{\circ}$ until ready for use. Aliquots were thawed, resuspended in $20 \%$ TCA, disrupted by bead beating at $4^{\circ}$ using $0.5 \mathrm{~mm}$ zirconia/silica or glass beads, and monitored microscopically until near complete disruption was observed. Samples were collected by centrifugation, then washed with $5 \%$ TCA, and the pellet was resuspended in $1 \times$ NuPAGE LDS Sample Loading Buffer (Life Technologies) with $100 \mathrm{mM}$ dithiothreitol (DTT). Samples were separated on $12 \%$ bisTris NuPAGE gels in $1 \times$ MOPS or MES running buffer (Life Technologies) or 15\% Laemmli gels (Laemmli 1970). Proteins were blotted to polyvinyl difluoride (PVDF) membranes by semidry electrophoretic transfer using the iBlot system (Life Technologies) or in Tris-glycine (25 mM Tris base, $192 \mathrm{mM}$ glycine, $10 \%$ methanol, $0.04 \%$ SDS) at $100 \mathrm{~mA}$ constant for 70 min (TransBlot SD Transfer Cell; Bio-Rad Laboratories, Hercules, CA). Membranes were air dried and then incubated with one of the following rabbit primary antibodies diluted in 5\% nonfat milk (NFM) in Tris-buffered saline (TBS)/Tween 20 buffer (TBST) (25 mM Tris-HCl, $\mathrm{pH}$ 7.4, $137 \mathrm{mM} \mathrm{NaCl}, 2.7 \mathrm{mM} \mathrm{KCl}, 0.1 \%$ Tween 20): antiH3 polyclonal (Abcam 1791) diluted 1:10,000, anti-H3 T11ph monoclonal (EMD Millipore 05-789) diluted 1:1000, anti-H3 T11ph polyclonal (Active Motif 39151) diluted 1:1000, anti-H3 S10ph monoclonal (EMD Millipore 05-817) diluted 1:1000, anti-H3 S10ph polyclonal (EMD Millipore 06-560) diluted 1:1000, or anti-H2A S129ph polyclonal (Abcam 15083) diluted 1:500. The polyclonal secondary antibody used was horseradish peroxidase-conjugated goat anti-rabbit (Pierce/Thermo Fisher Scientific 31462 or
31460) diluted 1:10,000 in TBST with visualization by the ECL-Plus kit (GE Healthcare) exposed to chemiluminescent film or charged-coupled device camera (Image Station; Eastman Kodak, Rochester, NY).

\section{Validation of anti-phospho-H3 antibodies}

Two commercial anti-H3 T11ph antibodies yielded Spo11dependent bands at the expected size for $\mathrm{H} 3$ on western blots, but the $\mathrm{mAb}$ gave a more robust signal with less background (Figure 1B). To more definitively characterize the specificity of these antibodies, we incubated them with synthetic peptide arrays containing different $\mathrm{H} 3$ modification states (Active Motif MODified Histone Peptide Array) (Table S2). The monoclonal anti-H3 T11ph antibody reacted strongly with all peptides containing T11ph regardless of other modifications present, unless S10 was also phosphorylated, in which case reactivity was strongly or completely lost (Figure S1Ai). This mAb was highly specific as little to no cross-reactivity was observed for unmodified H3 peptides; H3 peptides carrying other modifications; or peptides from other histones, including peptides phosphorylated at other sites (H3 S10ph, H3 S28ph, H4 S1ph, H2A S1ph, and H2B S14ph) (Figure S1Ai). In a more limited analysis, the polyclonal anti-H3 T11ph antibody bound specifically to a peptide with trimethylated $\mathrm{H} 3$ K9 (K9me3) as well as T11ph, but not to unmodified or S10ph peptides from $\mathrm{H} 3$ or full-length unmodified histones (Figure S1B). However, this polyclonal antibody (pAb) showed substantial nonhistone cross-reactivity against yeast whole-cell extracts (WCEs) that was not observed for the monoclonal anti-H3 T11ph antibody (Figure 1B).

Both the monoclonal and the polyclonal anti-H3 S10ph antibodies that we used reacted with phospho-S10 H3 peptide on dot blots, but with some background signal for full-length histone H3 (Figure S1B). Similarly, the polyclonal anti-H3 S10ph antibody detected S10ph on the peptide array, including in the context of other nearby modifications, unless T11 was also phosphorylated (Figure S1Aii). Again, however, modest cross-reactivity was seen with other histone $\mathrm{H} 3$ and H4 peptides, thus the anti-S10ph antibodies are less specific than the monoclonal anti-T11ph antibody.

\section{In vitro kinase assays}

GST-Mek1 and GST-mek1-as were affinity purified on glutathione sepharose as described (Niu et al. 2009; Lo and Hollingsworth 2011).

Radiolabeling method: Reactions included $2 \mu \mathrm{g}$ of recombinant $S$. cerevisiae histone H3 or $5 \mu \mathrm{g}$ H3 1-20 peptides, $250 \mathrm{ng}$ GST-Mek1, $0.4 \mathrm{mM}$ ATP, and $10 \mu \mathrm{Ci}\left[\gamma^{-32} \mathrm{P}\right]$-ATP $(6000 \mathrm{Ci} / \mathrm{mmol}$; PerkinElmer-Cetus, Norwalk, CT) in a $25-\mu$ l total volume in a buffer containing $50 \mathrm{mM}$ HEPES$\mathrm{NaOH}, \mathrm{pH} 7.5,150 \mathrm{mM} \mathrm{NaCl}, 10 \mathrm{mM} \mathrm{MgCl}_{2}, 0.5 \mathrm{mM}$ DTT, and $1 \times$ each of Roche phosphatase and protease inhibitor cocktails. Reactions were incubated at $30^{\circ}$ for $30 \mathrm{~min}$, resolved on $12 \%$ bis-Tris NuPAGE gels in $1 \times$ MES running buffer, and then transferred to PVDF via the iBlot system or 


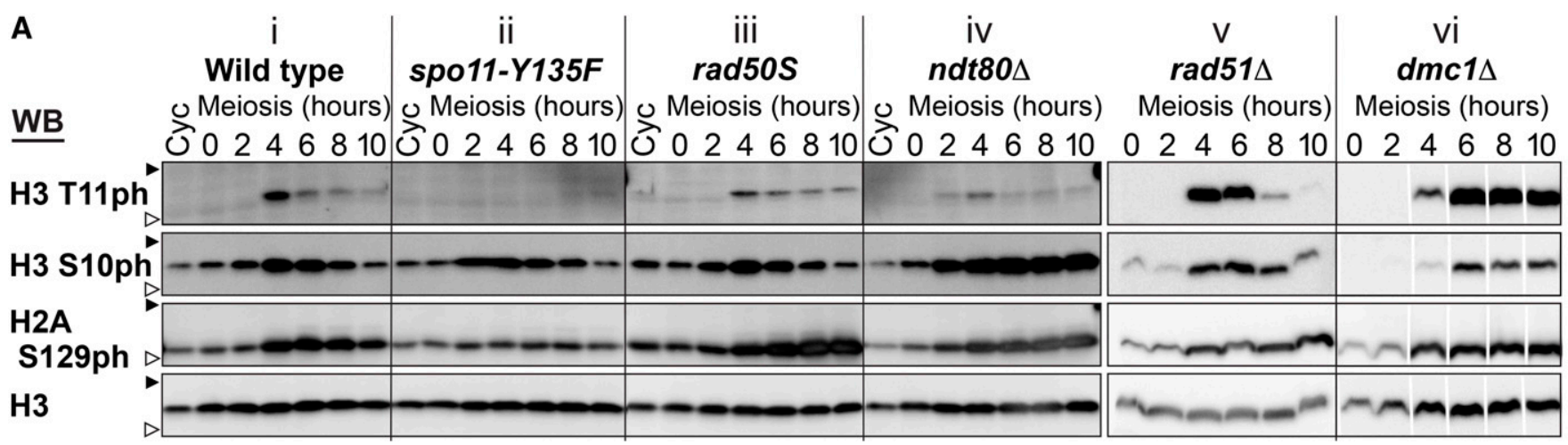

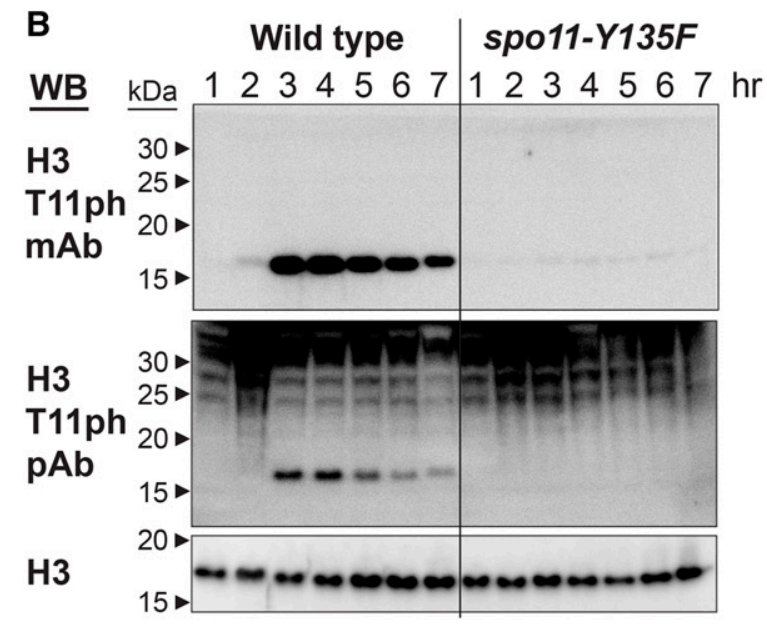

D

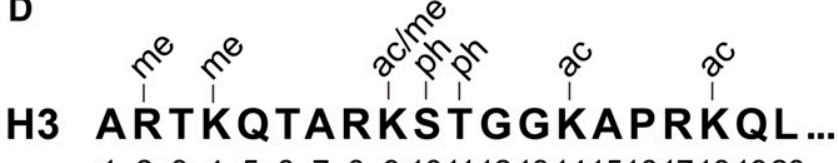

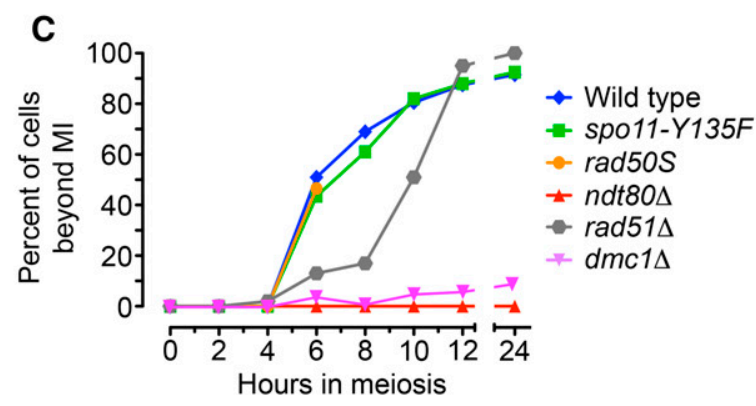

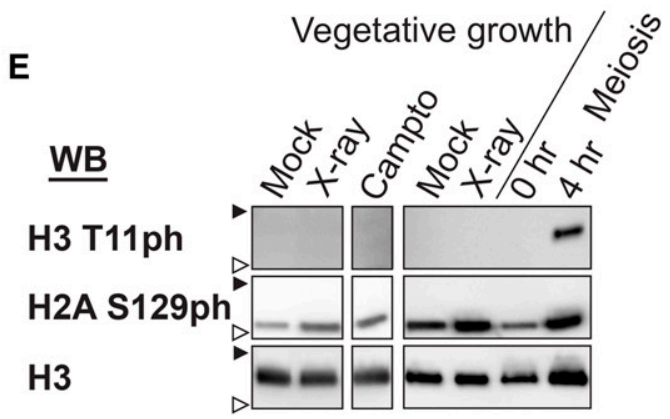

Figure 1 H3 T11 phosphorylation in S. cerevisiae meiosis. (A) Western blots of WCEs from asynchronous cycling vegetative (Cyc) and synchronized meiotic culture time points in wild-type and mutant strains. In (i-iv), the antibodies used were anti-H3 T11ph polyclonal (Active Motif 39151), anti-H3 S10ph monoclonal (EMD Millipore 05-817), anti-H2A S129ph/ $\gamma-\mathrm{H} 2 \mathrm{~A}$ (Abcam 15083), and anti-H3 (Abcam 1791). For (v and vi), anti-H3 T11ph monoclonal (EMD Millipore 05-789) and anti-H3 S10ph polyclonal (EMD Millipore 06-560) were used; other antibodies were the same. Interstitial lanes were removed from the blot images in (vi) to match time points in other panels. $\mathbf{\Delta}$ and $\triangle$ indicate 20- and 15-kDa molecular weight markers, respectively. (B) Western blot comparison of anti-H3 T11ph mAb (EMD Millipore 05-789) and pAb (Active Motif 39151). (C) Meiotic progression assessed by DAPI staining. Cells with two or more DAPI-staining bodies were scored as having progressed past the first meiotic division; $n \geq 100$ cells per time point. The rad50S culture was not quantified past $6 \mathrm{hr}$ because of nuclear fragmentation. (D) The first 20 amino acids in histone H3 and modifications known to occur in S. cerevisiae or S. pombe, with T11 underlined. (E) Meiosis-specificity of DNA damage-induced H3 T11ph. Asynchronous vegetative cultures of wild type were treated with genotoxins that induce DSBs and then WCEs were prepared and analyzed by western blotting for H3 T1 1 ph. Cultures in the left panel were untreated (Mock) or treated with X rays (400 Gy) or camptothecin (Campto) (20 $\mu$ M) at room temperature. An interstitial lane was deleted from the blot image for this panel. Cultures in the right panel were untreated or treated with $\mathrm{X}$ rays (400 Gy) on ice. Premeiotic $(0 \mathrm{hr})$ and meiotic $(4 \mathrm{hr})$ cultures were included as controls. The anti-H3 T11 ph mAb (EMD Millipore 05-789) was used. $\boldsymbol{\Delta}$ and $\triangle$ are as defined in (A). ac, acetylation; me, methylation; ph, phosphorylation; WB, western blot.

Coomassie stained and dried for autoradiography on a Fujifilm FLA 7000. The primary antibody was rabbit anti-H3 T11ph polyclonal (Active Motif 39151) diluted 1:500, with secondary antibody and detection carried out as described above.

Semisynthetic epitope method: GST-Mek1-as target labeling and detection followed previously described methods (Niu et al. 2009; Lo and Hollingsworth 2011). Reactions included
$2 \mu \mathrm{g}$ of recombinant $S$. cerevisiae histone H3, $2 \mu \mathrm{g}$ GST-Mek1 or $0.76 \mu \mathrm{g}$ GST-Mek1-as, $0.4 \mathrm{mM} \mathrm{ATP} \gamma \mathrm{S}$ or 6-Fu-ATP $\gamma \mathrm{S}$ ( $\mathrm{N}^{6}$-furfuryladenosine-5'-O-3-thiotriphosphate; Axxora), and $0.2 \mathrm{mM}$ ATP in a $25-\mu \mathrm{l}$ total volume in a buffer containing $50 \mathrm{mM}$ Tris-HCl, pH 7.5, $150 \mathrm{mM} \mathrm{NaCl}, 10 \mathrm{mM} \mathrm{MgCl}_{2}$, and $0.5 \mathrm{mM}$ DTT. Reactions were incubated at $30^{\circ}$ for $30 \mathrm{~min}$, p-nitrobenzyl mesylate (PNBM) (in DMSO; Abcam/Epitomics $3700-1$ ) was added to $2.5 \mathrm{mM}$, and then they were incubated at room temperature for $90 \mathrm{~min}$. Samples were electrophoresed 
on $4-12 \%$ bis-Tris NuPAGE gels in $1 \times$ MES running buffer, followed by semidry transfer to PVDF at $25 \mathrm{~V}$ constant for $60 \mathrm{~min}$. Membranes were blocked in 5\% NFM-TBST, primary antibodies were rabbit anti-thiophosphate ester monoclonal (Abcam/Epitomics 2686-1) diluted 1:5000 or rabbit anti-H3 T11ph monoclonal (EMD Millipore 05-789) diluted 1:1000, with secondary antibody and detection carried out as described above.

\section{Histone ChIP-sequencing}

The ChIP-sequencing (ChIP-seq) protocol was based on a previously described method (Wal and Pugh 2012). Two independent wild-type (SKY165) and one spo11-Y135F (SKY198) meiotic cultures were prepared as described (Murakami and Keeney 2014) and $4 \times 10^{9}$ cells were harvested at 3 and $4 \mathrm{hr}$ (wild type) and $3.5 \mathrm{hr}$ (spo11-Y135F) after the meiosis induction. Cells were fixed with $1 \%$ formaldehyde for $15 \mathrm{~min}$ at room temperature, with mixing at $50 \mathrm{rpm}$. Cross-linking was quenched by adding glycine to $131 \mathrm{mM}$ for $5 \mathrm{~min}$, cells were washed with water, resuspended in ice-cold ST buffer (10 mM Tris-HCl, pH 7.4, $100 \mathrm{mM} \mathrm{NaCl}$, and $1 \times$ each of Roche phosphatase and protease inhibitor cocktails). To compare different samples, we used $S$. pombe cells as a spike-in control. $S$. pombe cells (SKY2594) harvested at $4.5 \mathrm{hr}$ in meiosis were fixed and washed with the same condition described above. An aliquot of $4 \times 10^{7} \mathrm{~S}$. pombe cells ( $1 \%$ of the number of $S$. cerevisiae cells) were added to each sample.

Cells were resuspended in FA lysis buffer (50 mM HEPES$\mathrm{NaOH}$, pH 7.5; 150 mM NaCl; 2 mM EDTA; 1\% Triton X-100; $0.1 \%$ sodium deoxycholate; $10 \mu \mathrm{g} / \mathrm{ml}$ each of leupeptin, pepstatin A, and chymostatin; $1 \mathrm{mM}$ PMSF; and $1 \times$ each of Roche phosphatase and protease inhibitor cocktails) and disrupted using zirconia/silica beads $(0.5 \mathrm{~mm}$, Biospec Products 11079105z) and a FastPrep-24 (MP Biomedicals) with eight rounds of shaking at $6.5 \mathrm{~m} / \mathrm{sec}$ for $60 \mathrm{sec}$. Lysates were pelleted by centrifugation at 15,000 rpm for $5 \mathrm{~min}$ at $4^{\circ}$, washed with NP-S buffer (0.5 mM spermidine; 0.075\% IGEPAL CA-630; $50 \mathrm{mM} \mathrm{NaCl} ; 10 \mathrm{mM}$ Tris-HCl, pH 7.4; $10 \mathrm{mM}$ $\mathrm{MgCl}_{2} ; 2 \mathrm{mM} \mathrm{CaCl} ; 10 \mu \mathrm{g} / \mathrm{ml}$ each of leupeptin, pepstatin $\mathrm{A}$, and chymostatin; $1 \mathrm{mM}$ PMSF; $1 \times$ each of Roche phosphatase and protease inhibitor cocktails) and resuspended in $3.6 \mathrm{ml}$ NP-S buffer with $1 \mathrm{mM}$ 2-mercaptoethanol. The resuspended pellet (chromatin) was solubilized by digestion with 25 units/ml of micrococcal nuclease (Worthington Biochemical, Freehold, NJ) at $37^{\circ}$ for $20 \mathrm{~min}$. Digestion was terminated by adding EDTA to $10 \mu \mathrm{M}$ and SDS to $0.05 \%$. Chromatin was further solubilized by sonication (Biorupter Standard, Diagenode) on the highest setting for two rounds of $30 \mathrm{sec}$ with a $30 \mathrm{sec}$ intervening rest. Solubilized chromatin was isolated by centrifugation at $16,000 \mathrm{rpm}$ for $10 \mathrm{~min}$ at $4^{\circ}$, pooled, and divided into two equal volumes.

A total of $20 \mu \mathrm{g}$ of each antibody was added to the micrococcal nuclease (MNase)-treated chromatin samples and incubated at $4^{\circ}$ overnight on a rotisserie mixer [antibodies: rabbit anti-H3 pAb (Abcam 1791) and rabbit anti-H3
T11ph mAb (EMD Millipore 04-789)]. Immunoprecipitation was carried out by adding $200 \mu \mathrm{l}$ protein G Dynabeads (Life Technologies) and incubating at $4^{\circ}$ for $90 \mathrm{~min}$ on a rotisserie mixer. Beads were washed with $1 \mathrm{ml}$ of the following buffers: NP-S buffer, FA lysis buffer, $2 \times$ FA high salt buffer (FA lysis buffer containing $1 \mathrm{M} \mathrm{NaCl}$ ), $2 \times$ FA wash 2 buffer (FA lysis buffer containing $0.5 \mathrm{M} \mathrm{NaCl}$ ), $2 \times$ FA wash 3 buffer $(10 \mathrm{mM}$ Tris-HCl, pH 8, 250 mM LiCl, 2 mM EDTA, 1\% IGEPAL, 1\% sodium deoxycholate), and TE wash buffer (10 mM Tris- $\mathrm{HCl}$, pH 8, 1 mM EDTA, 0.5\% Triton X-100). Bound nucleosomes were eluted, reverse cross-linked, and treated with RNase $\mathrm{A}$ and Proteinase $\mathrm{K}$ as described (Murakami and Keeney 2014). DNA was purified using a PCR purification kit (QIAGEN, Valencia, CA) and separated on a 1.5\% agarose gel. Mononucleosome-sized DNA ( $\sim 150 \mathrm{bp}$ ) was extracted from the gel and prepared for 50-nt paired-end sequencing on the Hiseq platform (Illumina) following standard Illumina protocols. Sequencing was performed at the Integrated Genomics Operation of the Memorial Sloan Kettering Cancer Center.

Paired-end 50-nt reads were mapped to the $S$. cerevisiae reference genome (sacCer2) and the Sanger Center's $S$. pombe genome version of August 7, 2010 using BWA (version 0.7.12r1039) MEM (Li 2013). Paired reads with an insert size $>250$ bp were filtered out and the rest were converted into coverage maps. All downstream analyses were carried out using R (http://www.r-project.org/) (R Development Core Team 2012). Each coverage map was normalized per 1000 reads from $S$. pombe chromosomes I and II. Cumulative S1 sequencing ( $\mathrm{S} 1$-seq) values were generated by calculating the cumulative sum of the top strand (or bottom strand) reads of S1-seq data (Mimitou et al. 2017) from the midpoint of each hotspot to $2 \mathrm{~kb}$ downstream (or upstream).

\section{Red1 ChIP-seq}

The Red1 ChIP-seq protocol was based on a previously described method (Kugou et al. 2009). Meiotic cells $\left(5 \times 10^{8}\right)$ expressing FLAG-tagged Red1 (strain YKT190) were harvested at $3 \mathrm{hr}$ after the meiosis induction. Cells were fixed with $1 \%$ formaldehyde for $10 \mathrm{~min}$ at room temperature, and cross-linking was quenched by adding glycine to $125 \mathrm{mM}$ for 5 min. Cells were washed with ice-cold TBS (20 mM Tris$\mathrm{HCl}, \mathrm{pH}$ 7.6, $150 \mathrm{mM} \mathrm{NaCl}$ ).

Cells were resuspended in lysis 140 buffer (50 mM HEPES$\mathrm{KOH}, \mathrm{pH} 7.5,140 \mathrm{mM} \mathrm{NaCl}, 1$ mM EDTA, pH 8.0, 1\% Triton $\mathrm{X}-100,0.1 \%$ sodium deoxycholate, $1 \times$ Roche protease inhibitor cocktail) and disrupted using zirconia beads $(0.5 \mathrm{~mm}$, Yasui Kikai) and a Multi-Beads Shocker (Yasui Kikai) with four rounds of shaking at $2300 \mathrm{rpm}$ for $60 \mathrm{sec}$ at $4^{\circ}$. Lysates were sonicated using Covaris to obtain genomic DNA fragments averaging $300 \mathrm{bp}$ and the supernatant (WCE) was collected after centrifugation at $15,000 \mathrm{rpm}$ for $15 \mathrm{~min}$ at $4^{\circ}$.

Immunoprecipitation was carried out by adding $3 \mu \mathrm{l}$ of anti-FLAG antibody (Wako) coupled to $80 \mu \mathrm{l}$ of protein A Dynabeads (Life Technologies) and incubating at $4^{\circ}$ for $3.5 \mathrm{hr}$ by rotating. Beads were washed with $1 \mathrm{ml}$ of the following buffers: lysis 140 buffer twice, lysis 500 buffer (50 mM 
HEPES-KOH, pH 7.5, 500 mM NaCl, 1 mM EDTA, pH 8.0, 1\% Triton X-100, 0.1\% sodium deoxycholate) once, LiCl/detergent buffer (10 mM Tris-HCl, pH 8.0, $250 \mathrm{mM} \mathrm{LiCl,} 1 \mathrm{mM}$ EDTA, pH 8.0, 0.5\% Nonident P-40, 0.5\% sodium deoxycholate) twice, and TE (10 mM Tris-HCl, pH 8.0, 1 mM EDTA, $\mathrm{pH}$ 8.0) once. Bead-bound chromatin was eluted in $100 \mu \mathrm{l}$ of TE/ 1\% SDS (50 mM Tris-HCl, pH 8.0, 10 mM EDTA, pH 8.0, 1\% SDS) by incubating at $65^{\circ}$ for $15 \mathrm{~min}$ and then in $150 \mu \mathrm{l}$ of TE/ 0.67\% SDS (50 mM Tris-HCl, pH 8.0, 10 mM EDTA, pH 8.0, $0.67 \%$ SDS) by incubating at $65^{\circ}$ for $5 \mathrm{~min}$. For input, a small volume of WCE was mixed with TE/1.33\% SDS (50 mM Tris$\mathrm{HCl}, \mathrm{pH}$ 8.0, 10 mM EDTA, pH 8.0, 1.33\% SDS). Cross-links in eluted chromatin and input DNA were reversed by incubating at $65^{\circ}$ overnight and treating with $8.4 \mu \mathrm{l}$ of proteinase $\mathrm{K}$ (Merck) at $50^{\circ}$ for $2 \mathrm{hr}$. DNA was purified using a PCR purification kit (QIAGEN) and further sonicated by Covaris to obtain DNA fragments averaging 150 bp. Multiplexed DNA libraries were prepared with NEBNext ChIP-Seq Library Prep Master Mix Set for Illumina (NEB) and NEBNext Multiplex Oligos for Illumina (NEB). ChIP and input DNA were sequenced by Illumina Misequation (four samples per run, $50 \mathrm{nt}$ single-end read) using MiSeq Reagent Kit v2.

Single-end 50-nt reads were filtered and end trimmed, followed by removal of the reads containing tag sequences, and then mapped to the $S$. cerevisiae reference genome (sacCer2) as described (Ito et al. 2014). DNA enrichment (ChIP/input) was calculated in 10-bp bins with normalization as described (Ito et al. 2014). A total of 1717 Red 1 peaks were called as described (Murakami and Keeney 2014) using a 2010-bp Parzen (triangular) sliding window and a threshold of $1 \times$ genomic mean coverage.

\section{Data availability}

Plasmids and strains are available on request. ChIP-seq data are available at the Gene Expression Omnibus under accession numbers GSE100564 (H3 and H3 T11ph) and GSE103823 (Red1).

\section{Results}

\section{H3 T11 phosphorylation during meiosis is a response to DSBS}

As part of a larger effort to identify meiotically regulated histone modifications in $S$. cerevisiae, we performed western blots on meiotic WCEs with antibodies to H3 T11ph. Under these conditions, signal was undetectable in mitotically cycling, premeiotic $\left(\mathrm{G}_{1}\right.$ arrested, $0 \mathrm{hr}$ ), or early meiotic (through $2 \mathrm{hr}$ ) cultures, but accumulated transiently during meiosis with a maximum at $\sim 3-5 \mathrm{hr}$ (Figure 1 , Ai and B). This signal diminished as cells completed the first meiotic division ( $\sim 7$ hr; Figure 1, Ai, B, and C). These findings agreed with studies reported while this work was in progress (Govin et al. 2010).

The anti-H3 T11ph signal occurred when DSBs are usually maximal under these conditions [ 3-5 hr (e.g., Thacker et al. 2014)], and coincided with an increase in H2A S129 phos- phorylation $(\gamma-\mathrm{H} 2 \mathrm{~A})$ (Figure 1Ai), which is formed by Mec1 and Tel1 kinases in response to meiotic DSBs (Mahadevaiah et al. 2001; Shroff et al. 2004). These results suggested H3 T11ph might be a DSB response, but H3 T11ph signal also coincided with an increase in H3 S10 phosphorylation (Figure 1Ai), which is DSB independent (Hsu et al. 2000).

We therefore examined the genetic requirements for $\mathrm{H} 3$ T11ph. The modification was undetectable in a strain with catalytically inactive Spo11 (spo11-Y135F; Figure 1, Aii and B). As expected, induction of higher $\gamma$-H2A signal was not seen in spo11-Y135F, but H3 S10ph was induced (Figure 1Aii). H3 T11ph appeared in a rad50S strain, in which DSBs form but persist with unresected $5^{\prime}$ ends, so DSB resection is dispensable (Figure 1Aiii). H3 S10ph was unaffected in this mutant, but elevated $\gamma$-H2A levels persisted to late time points, consistent with unmitigated Tel1 activity (Usui et al. 2001).

H3 T11ph appeared and disappeared in rad51s with kinetics similar to wild type (Figure 1Av), but persisted at high levels in $d m c 1 \Delta$ (Figure 1Avi) (a different antibody was used for these blots, discussed below). Both rad51 $1 \Delta$ and $d m c 1 \Delta$ have defects in meiotic DSB repair (note the persistent $\gamma$-H2A), but with a more complete block in $d m c 1 \Delta$ (Bishop et al. 1992; Shinohara et al. 1992). Meiotic arrest is also nearly complete in $d m c 1 \Delta$, whereas divisions occur in rad51 $\Delta$ after a delay (Figure 1C) (Bishop et al. 1992; Shinohara et al. 1992).

To determine whether this persistent H3 T11ph signal was due to persistent DSBs or to meiotic arrest, we examined an ndt80 $\Delta$ mutant. Ndt80 is a transcription factor needed for pachytene exit (Xu et al. 1995; Chu and Herskowitz 1998), and DSB repair defects cause arrest via checkpoint kinasemediated inhibition of Ndt80 (Tung et al. 2000; Gasior et al. 2001). H3 T11ph did not persist at high levels in an ndt80 8 mutant; instead it peaked at $4 \mathrm{hr}$ at a slightly lower level than in wild type, and then diminished to low residual levels similar to those seen at late times in wild type (Figure 1Aiv). This agrees with a recent report demonstrating $\mathrm{H} 3$ T11ph appearance and disappearance by western blotting and immunofluorescence of spread chromosomes (Subramanian et al. 2016). Therefore, high-level H3 T11ph persistence correlates with continued presence of meiotic DSBs (as in $d m c 1 \Delta$ ), but not with arrest. This behavior contrasts with that of a different Mek1 substrate, Hed1, which remains phosphorylated in ndt80s mutants (Prugar et al. 2017). Both $\gamma$-H2A and $\mathrm{H} 3$ $\mathrm{S} 10 \mathrm{ph}$ persisted at high levels in ndt80A (Figure 1Aiv) (Hsu et al. 2000), suggesting these modifications require pachytene exit for removal (Subramanian et al. 2016).

Because the $\mathrm{H} 3 \mathrm{~N}$-terminal tail has many potential modification sites (Figure 1D) and a different antibody not used in our studies cross-reacts between H3 T11ph, H3 S10ph, and other modifications (Nady et al. 2008); we sought to validate the antibody specificity for the anti-H3 T11ph antibodies we used. Both the monoclonal and polyclonal anti-H3 T11ph antibodies were specific but did not detect H3 T11ph if S10 was also phosphorylated (Figure S1 and Materials and Methods). 
A

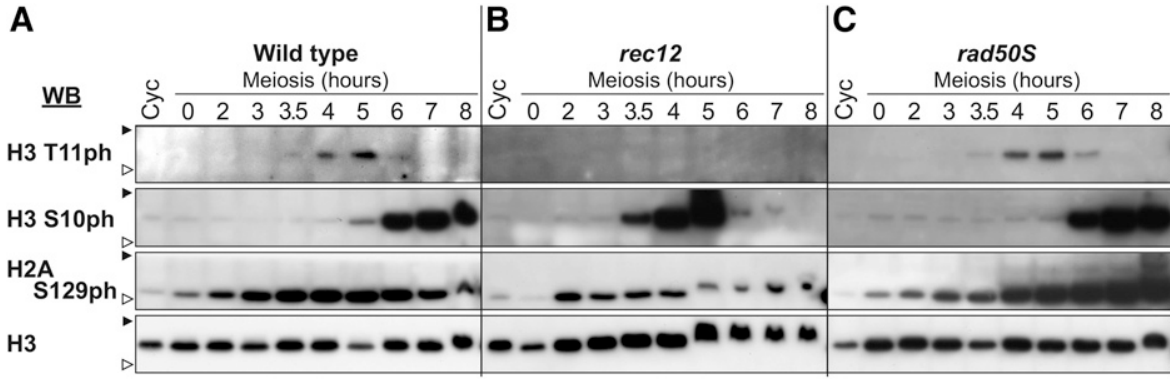

Figure 2 (A-C) H3 T11 phosphorylation in S. pombe meiosis. Western blots of WCEs from haploid pat1-114 strains undergoing synchronized meiosis. Antibodies used were the same as in Figure $1 \mathrm{Av}$. $\boldsymbol{\Delta}$ and $\triangle$ indicate 20 - and $15-\mathrm{kDa}$ molecular weight markers, respectively. The altered electrophoretic mobility of histones at later time points in some cultures was probably caused by varying levels of contaminating DNA in the extracts rather than differential post-translational modifications. Cyc, cycling vegetative; WB, western blot.
The $\mathrm{mAb}$ gave a more robust signal with less background from nonhistone proteins (Figure 1B), so we used this antibody for most subsequent experiments. Two different anti-H3 S10ph antibodies recognized their cognate modification, but not if T11 was also phosphorylated. These anti-H3 S10ph antibodies showed significant cross-reactivity to other histones and modifications (Figure S1 and Materials and Methods).

To test if DNA lesions could also give rise to elevated T11 phosphorylation during vegetative growth, cells were treated with $\mathrm{X}$ rays or camptothecin. These DNA damaging agents failed to yield a detectable level of $\mathrm{H} 3 \mathrm{~T} 11 \mathrm{ph}$, despite inducing DNA damage responses as evidenced by increased $\gamma$-H2A (Figure 1E). Thus, high levels of $\mathrm{H} 3 \mathrm{~T} 11 \mathrm{ph}$ in response to DNA damage are specific to meiosis. The strength of the meiotic H3 T11ph signal as compared to the undetectable levels under these blotting conditions for cycling or premeiotic cells, or the spo11-Y135F mutant, indicates that the amount of $\mathrm{H} 3 \mathrm{~T} 11 \mathrm{ph}$ formed in meiosis is vastly greater than what has been reported to be formed by pyruvate kinase during vegetative growth (Li et al. 2015).

\section{H3 T11ph in response to DSBs in S. pombe meiosis}

To determine if meiotic H3 T11ph is evolutionarily conserved, we analyzed synchronous meiosis in $S$. pombe haploid pat1114 mutants (Bahler et al. 1991). H3 T11ph appeared transiently at $\sim 4-5 \mathrm{hr}$ after the initiation of meiosis and was not detected in a mutant lacking Rec12 (the Spo11 ortholog) or in vegetative growth (Figure 2). H3 T11ph appeared after a Rec12-dependent increase in $\gamma$-H2A that started $\sim 3-3.5 \mathrm{hr}$, when DSBs typically appear under these conditions (Cervantes et al. 2000). [The initial wave of $\gamma$-H2A signal at or before $2 \mathrm{hr}$ is Rec12 independent (Figure 2B) and possibly associated with DNA replication.] These results indicate that $\mathrm{H} 3$ T11ph forms in response to DSBs in S. pombe. H3 T11ph appeared and disappeared with apparently normal kinetics in a rad50S mutant in contrast to $\gamma-\mathrm{H} 2 \mathrm{~A}$, which persisted at high levels (Figure 2C).

H3 S10ph also appeared during meiosis, but unlike in $S$. cerevisiae, this modification occurred later than $\mathrm{H} 3 \mathrm{~T} 11 \mathrm{ph}$ (Figure 2A). In the rec12 mutant, H3 S10ph was observed earlier than normal and was largely gone by $6 \mathrm{hr}$ (Figure 2B). This result is consistent with accelerated meiotic progression in rec12 mutants (Doll et al. 2008), and indicates that both the appearance and disappearance of H3 S10ph is developmentally regulated.

\section{H3 T11 is a direct target of Mek1 kinase}

The timing and genetic control of H3 T11ph in S. cerevisiae suggested that a meiosis-specific, DSB-responsive kinase was responsible. Mek1 expression coincides with $\mathrm{H} 3 \mathrm{~T} 11 \mathrm{ph}$ from 3 to $7 \mathrm{hr}$ in meiosis (Carballo et al. 2008), and the T11 sequence context matches the Mek1 target consensus (RXXT; Figure 1D) (Mok et al. 2010; Suhandynata et al. 2016). We therefore treated a $d m c 1 \Delta$ strain expressing an ATP analogsensitive mek1 allele (mek1-as) with an inhibitor specific for the mutated Mek1 kinase, 1-NA-PP1 (Wan et al. 2004). Inhibitor addition at $4 \mathrm{hr}$ caused rapid disappearance of $\mathrm{H} 3$ T11ph within the first hour (Figure 3A). This result demonstrates that Mek1 activity is necessary to maintain H3 T11 phosphorylation, and further implies that this modification is dynamic with a half-life much $<1 \mathrm{hr}$.

This result agreed with prior findings demonstrating that $\mathrm{H} 3 \mathrm{~T} 11 \mathrm{ph}$ is reduced or absent in a mek1 $\Delta$ mutant (Govin et al. 2010). However, these findings did not establish whether H3 T11 is a direct target of Mek1. To address this question, we carried out two types of in vitro kinase assays using GSTtagged Mek1 purified from meiotic $S$. cerevisiae cells (Wan et al. 2004; Niu et al. 2007). First, we used [ $\left.\gamma^{-32} \mathrm{P}\right]$ ATP and full-length $\mathrm{H} 3$ or synthetic $\mathrm{H} 3$ peptides as substrates (Figure 3B). GST-Mek1 was visible in all lanes by Coomassie staining (Figure 3B, bottom panel) and its activity was confirmed by its ability to autophosphorylate (Figure 3B, top panel) (Niu et al. 2009). GST-Mek1 was able to phosphorylate full-length H3 and a peptide representing $\mathrm{H} 3$ amino acids 1-20 (Figure 3B, top panel, lanes 2 and 3). Phospho-transfer was specific for T11, as shown by western blot (Figure 3B, middle panel, lanes 2 and 3) and the inability to label an H3 1-20 peptide that was already phosphorylated on T11 (Figure 3B, lane 5). Interestingly, GST-Mek1 was also unable to phosphorylate a peptide carrying a phosphate on S10 (Figure 3B, top panel, lane 4).

The second assay used a semisynthetic epitope system (Allen et al. 2007) to detect phosphorylation of H3 by Mek1. GST-Mek1 or GST-Mek1-as was incubated with recombinant $\mathrm{H} 3$ and the ATP $\gamma \mathrm{S}$ analog, 6-Fu-ATP $\gamma \mathrm{S}$. Thiophosphates transferred by Mek1 to substrates were then alkylated to create an epitope that was detected on western blots with an anti-thiophosphate ester antibody (Niu et al. 2009; Lo and Hollingsworth 2011). Both GST-Mek1 and GSTMek1-as exhibited autophosphorylation and phosphorylation of H3 (Figure 3C, lanes 2 and 5). Moreover, 1-NA-PP1 

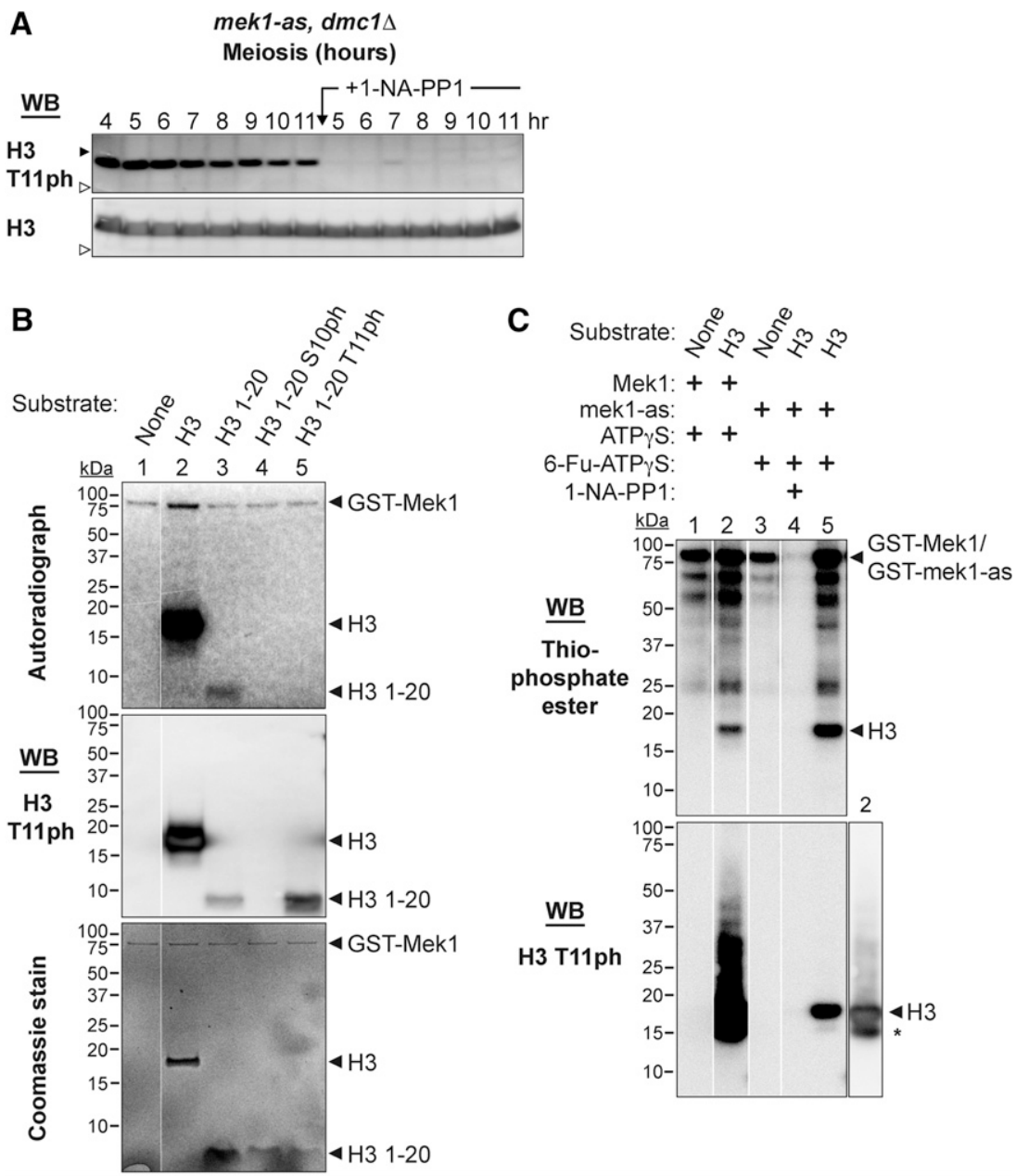

Figure 3 H3 T11 is a direct target of Mek1 kinase. (A) Persistence of $\mathrm{H} 3 \mathrm{~T} 11 \mathrm{ph}$ requires maintenance of Mek1 kinase activity. A meiotic culture of mek1-as, $d m c 1 \Delta$ (strain SKY3095) was split $4 \mathrm{hr}$ after transfer to sporulation medium. One part was left to continue in meiosis untreated, the other part was treated with $1 \mu \mathrm{M}$ 1-NA-PP1. WCEs were prepared at the indicated times and assayed for $\mathrm{H} 3 \mathrm{~T} 11 \mathrm{ph}$ by western blotting (mAb; EMD Millipore 05-789). $\Delta$ and $\triangle$ indicate 20 - and 15-kDa molecular weight markers, respectively. Numbers indicate hours after transfer to sporulation medium. (B) Mek1 kinase assay using radioactive ATP. Affinity-purified GST-Mek1 (250 ng) was incubated in the presence of [ $\gamma$-32P]ATP either alone or with $2 \mu \mathrm{g}$ recombinant $\mathrm{H} 3$ or $5 \mu \mathrm{g}$ of unphosphorylated or phosphorylated synthetic $\mathrm{H} 3$ 1-20 peptides as substrates. Reactions were separated by SDS-PAGE and visualized by autoradiography (top), anti-H3 T11ph western blot (middle; polyclonal Active Motif 39151), and Coomassie staining. (C) Mek1 kinase assay by semisynthetic epitope labeling. Kinase reactions were carried out with affinity-purified GST-Mek1 $(2 \mu \mathrm{g})$ or GST-Mek1-as $(0.76 \mu \mathrm{g})$ in the presence of ATP $\gamma S$ or 6-Fu-ATP $\gamma S$ with $2 \mu \mathrm{g}$ recombinant $\mathrm{H} 3$. After incubation $30 \mathrm{~min}$ at $30^{\circ}$, PNBM was added to alkylate the thiophosphorylated target sites. Reactions were then separated by SDS-PAGE and analyzed by western blotting with anti-thiophosphate ester mAb (top panel; Epitomics 2686-1). Because ATP was also included in all reactions, samples were also analyzed by western blotting with anti-H3 T11 ph $\mathrm{mAb}$ (EMD Millipore 05-789) to detect the subset of $\mathrm{H} 3$ proteins modified on T11 with phosphate instead of thiophosphate. Note that 1-NA-PP1 inhibits ability of GST-Mek1-as1 to use either ATP or Fu-ATP $y$ S to phosphorylate H3 T11 (lane 4). A shorter exposure of lane 2 of the anti-H3 T11ph blot is shown to the right (* indicates an $\mathrm{H} 3$ fragment present in the recombinant histone sample). Interstitial lanes were removed from images in ( $B$ and $C$ ) as indicated by the white lines. WB, western blot. inhibited both autophosphorylation and $\mathrm{H} 3$ phosphorylation by GST-Mek1-as (Figure 3C, lane 4), ruling out the possibility of a contaminating kinase phosphorylating H3 T11. We conclude that H3 T11 is a direct substrate of Mek1.

\section{Limitations of a plasmid shuffle system for examining histone mutants}

To determine the function of H3 T11 phosphorylation, we constructed strains carrying targeted mutations of T11 alone and in combination with other histone mutations. We initially tested an existing plasmid shuffle system (Ahn et al. 2005) by porting it to the SK1 strain background. In this approach, also used independently by others (Govin et al. 2010), the endogenous histone genes were deleted and complemented by wild-type histone genes on a URA3-marked ARS-CEN plasmid. Histone mutants were introduced on a separate LEU2 ARS-CEN plasmid and loss of the URA3 plasmid was selected for on a medium containing 5-FOA.

However, this approach was suboptimal because of the poor stability of the ARS-CEN plasmids in SK1. For example, when liquid cultures of the base histone-deletion strain carrying the URA3-covering plasmid were grown under conditions selective for the plasmid (i.e., synthetic complete medium lacking uracil), plating on solid medium yielded an efficiency of only $67.2 \pm 4.9 \%$ (mean \pm SD of five replicates; colony-forming units per cell plated). Assuming that most cells that failed to form a colony were those that had lost the plasmid because of missegregation during mitosis, it is likely that plasmid copy number per cell is highly variable in the population. Cells with one $v s$. two copies of an $\mathrm{H} 3 / \mathrm{H} 4-$ encoding plasmid would likely differ in total histone protein levels and/or have different imbalances with endogenous $\mathrm{H} 2 \mathrm{~A} / \mathrm{H} 2 \mathrm{~B}$. Altered histone gene dosage can cause deleterious effects (Meeks-Wagner and Hartwell 1986; Clark-Adams et al. 1988), so it is possible that cell-to-cell heterogeneity in histone gene copy number might mask or exacerbate the effects of histone point mutations. Furthermore, differences in copy number might have a substantial effect on variation in viability of spores (see below). Finally, although cells in the culture that have lost the histone plasmid would be inviable 
and therefore presumably would not sporulate, they would contribute to population average measurements in physical assays of recombination.

To circumvent these limitations, we turned to mutagenesis methods that use gene replacement or stable chromosomal integration (Materials and Methods). Stable integration is relatively rapid and obviates concerns about plasmid stability and heterogeneous gene dosage, but may not fully recapitulate expression from endogenous histone gene loci. The gene replacement strategy provides an even cleaner manipulation of histone genotype, but is more cumbersome because it requires separately mutating two histone gene loci.

\section{Absence of H3 T11 phosphorylation causes little or no overt phenotypes by itself}

We replaced both endogenous H3 genes (HHT1 and HHT2) with $h$ ht1-S10A, T11V and $h h t 2-S 10 A, T 11 \mathrm{~V}$ mutant alleles to eliminate phosphorylation of both $\mathrm{S} 10$ and T11. This mutant expressed normal $\mathrm{H} 3$ protein levels and neither $\mathrm{H} 3 \mathrm{~S} 10 \mathrm{ph}$ nor H3 T11ph could be detected, as expected (Figure 4A, lanes 3-4). The mutant displayed normal vegetative growth (Figure 4B), similar to a recent report (Li et al. 2015). Surprisingly, however, the mutant also displayed normal spore viability (Table 1). Meiotic DSBs appeared in normal numbers and locations and disappeared with normal kinetics as assessed by Southern blotting of pulsed-field gels probed for chromosome III (Figure 4C), and meiotic progression was not delayed (Figure 4D). These results indicate that most if not all meiotic events occur efficiently in the complete absence of both S10ph and T11ph.

To more easily manipulate histone mutants, we used a chromosomal integration strategy to introduce genes for just H3 and H4 as a pair (HHT2-HHF2) or all four core histones (HTA1-HTB1, HHT2-HHF2) in strains deleted for the endogenous genes for $\mathrm{H3}-\mathrm{H} 4$ or all four histones. Wild-type or mutant histone genes were integrated on chromosome III at LEU2. Strains expressing H3-S10A, -T11V, or -T11A single mutant proteins or the H3-S10A T11V double mutant were examined in meiotic time courses for H3 S10 and T11 phosphorylation (Figure 4A). Importantly, H3 T11 could still be phosphorylated when S10 was mutated to alanine (Figure 4A, lanes 9-12); the lower signal in the anti-H3 T11ph western blot could reflect reduced T11 phosphorylation or decreased antibody affinity due to the changed epitope. Similarly, mutation of H3 T11 to alanine or valine did not prevent phosphorylation of S10, as detected with the polyclonal anti-H3 S10ph antibody, although recognition by the monoclonal anti-H3 S10ph antibody was sensitive to these mutations (Figure 4A, lanes 13-18 and 21-22).

As with gene replacement, all of these mutants yielded spore viabilities indistinguishable from matched wild-type controls (Table 1). Meiotic divisions were not delayed; if anything, divisions were slightly earlier in the $H 3$ mutants than in the control strain (Figure 4D). Whether this difference has functional significance is unclear, especially given that H3-T11 mutations did not alleviate arrest caused by DSB repair defects (see below). H3-T11A also supported wild-type interhomolog recombination between arg4 heteroalleles [23 \pm $1.5 \mathrm{Arg}^{+}$recombinants per 1000 viable cells for wild type (SKY3428) vs. $24 \pm 0.8$ for H3-T11A (SKY3431), mean \pm SD for three independent cultures]. Other mutations of H3 T11 yielded similar results: changing T11 to serine or potential phospho-mimetic residues (T11D or T11E) again yielded wild-type spore viability (Table 1). Mutating H3 T11 also did not reduce spore viability when combined with mutation of H2A S129 [which is also by itself largely dispensable for proper meiosis (Shroff et al. 2004; Harvey et al. 2005)] or with absence of the H3 K4 methyltransferase Set1 [which governs DSB distributions (Sollier et al. 2004; Borde et al. 2009; Acquaviva et al. 2013; Sommermeyer et al. 2013)] (Figure 4A, lanes 21-22, and Table 2).

Mek1 is required for arrest or delay of meiotic progression when recombination is defective (Xu et al. 1997; Bailis and Roeder 2000). If $\mathrm{H} 3 \mathrm{~T} 11 \mathrm{ph}$ contributes substantially to this Mek1 function, then T11 mutations should alleviate some or all of the meiotic block in rad51s or dmc1s mutants. However, in cells lacking Rad51, the H3-T11V mutation had negligible effect on either the timing or efficiency of meiotic divisions (Figure 4E) and failed to rescue the spore inviability (Table 2). This H3 mutation also failed to alleviate the more stringent arrest in a $d m c 1 \Delta$ mutant (Figure 4E). Thus, H3 $\mathrm{T} 11 \mathrm{ph}$ is dispensable for this checkpoint arrest function of Mek1.

Our findings differ from a prior report of an $\sim 35 \%$ decrease in spore viability with plasmid-borne $H 3-T 11 A$ single or $S 10 A$ T11A double mutants (Govin et al. 2010). We obtained the published H3-T11A plasmid and histone-deleted SK1 host strain (generously provided by J. Govin and S. Berger), verified the H3-T11A mutation by sequencing, and carried out the plasmid shuffle. Three independent 5-FOA-resistant clones for each genotype were sporulated and tetrads were dissected for wild type and H3-T11A side by side. The experiment was repeated three times by two investigators. In our hands, this H3-T11A mutant again yielded spore viability indistinguishable from the control with a wild-type $H 3$ plasmid (Figure 4F and Table 1; $P>0.9$ by linear regression). However, unlike the normal spore viability observed in the stable integrant and gene replacement strains (Table 1), viability was consistently lower with plasmid-borne histone genes regardless of the $H 3$ genotype (Figure $4 \mathrm{~F}$ and Table 1 ). A similar baseline defect was reported previously (Govin et al. 2010). Furthermore, there was substantial heterogeneity in viability from experiment to experiment and between clones within each experiment (Figure 4F and Table 1). Within-experiment heterogeneity likely reflects stochastic culture-to-culture variability caused by plasmid instability. Between-experiment variability may reflect differences in sporulation conditions that in turn affect plasmid stability or the sensitivity of these strains to alterations in histone gene expression.

As a counterexample, we also examined a more extreme H3 mutant in which the entire amino-terminal tail was deleted $(H 3-\Delta N)$. The truncated histone was expressed at levels 
A

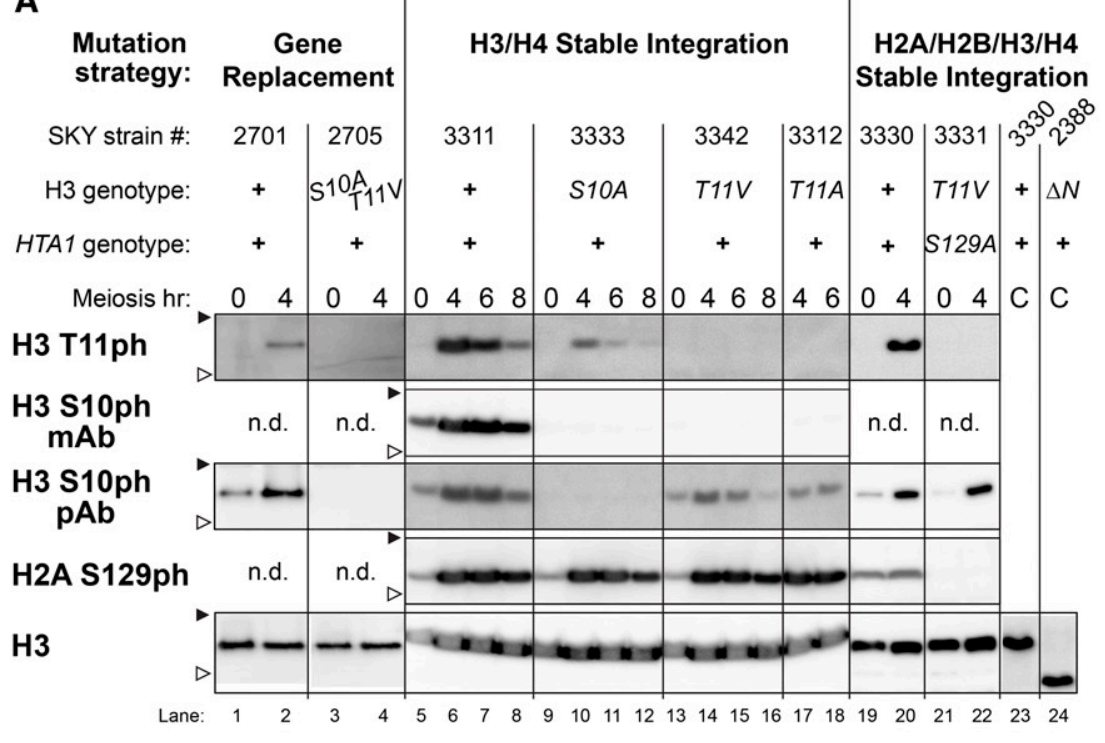

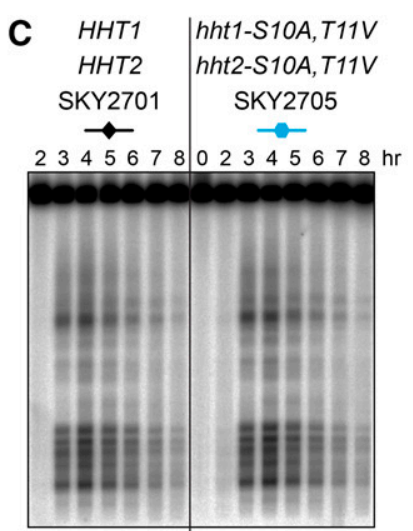

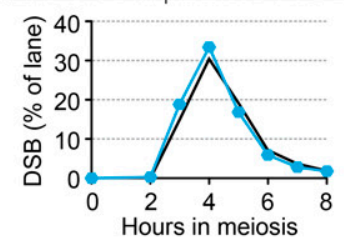

B

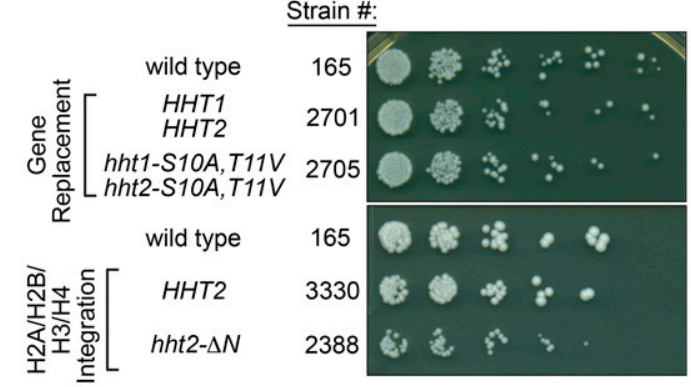

E

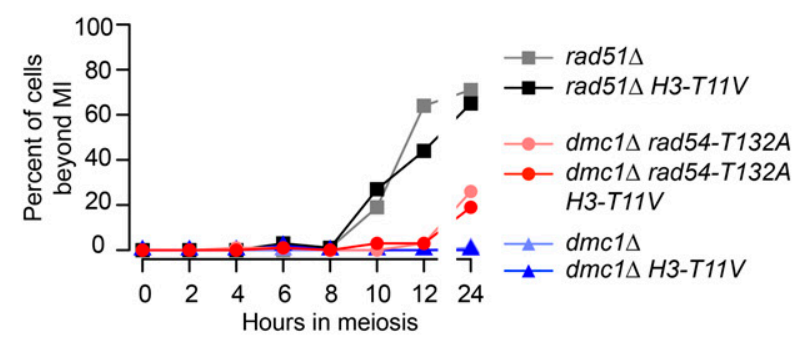

D

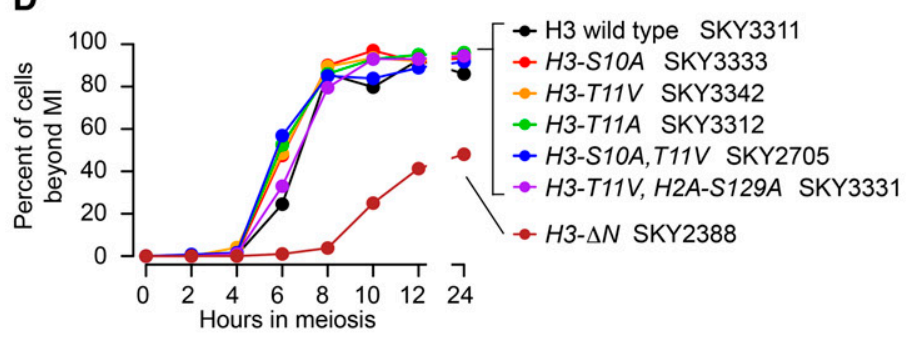

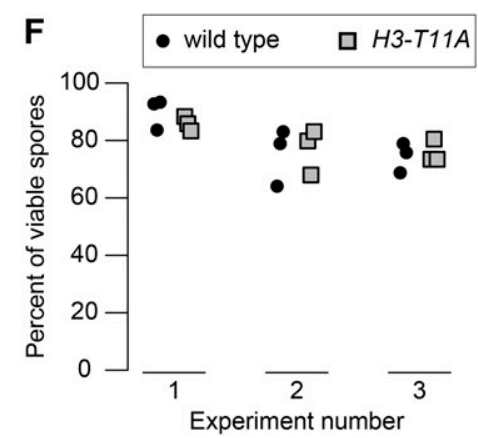

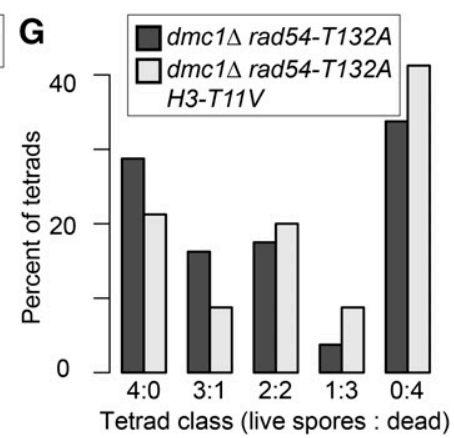

Figure 4 Characterization of histone mutant strains. (A) Composite of western blots of WCEs from synchronous meiotic cultures or asynchronous cycling vegetative cultures ("C") carrying the indicated histone mutations. Antibodies used were: anti-H3 T11ph polyclonal (Active Motif 39151) or antiH3 T11ph monoclonal (EMD Millipore 05-789), anti-H3 S10ph monoclonal (EMD Millipore 05-817), anti-H3 S10ph polyclonal (EMD Millipore 06-560), anti- $\gamma$-H2A (Abcam 15083), and anti-H3 (Abcam 1791). $\boldsymbol{\Delta}$ and $\triangle$ indicate 20- and 15-kDa molecular weight markers, respectively. (B) Vegetative growth of $\mathrm{H3}$ mutant strains. Cells from overnight cultures were spotted onto YPD plates using a manifold pin replicator and represent 1:5 serial dilutions starting with $\sim 2.5 \times 10^{6} \mathrm{cell} / \mathrm{s} / \mathrm{ml}$. (C) Analysis of meiotic DSB formation. High-molecular-weight DNA isolated in agarose plugs was separated by pulsed-field gel electrophoresis followed by Southern blotting and indirect end labeling with a probe directed against CHA1 on the left arm of chromosome III. The bottom panel shows quantification of the DSB signal as percent of lane total after background subtraction. (D and E) Meiotic progression of representative histone mutant strains. Cells were fixed and stained with DAPI and the fraction of cells with two or more nuclei was counted ( $n \geq 100$ cells per time point). For (D), points represent mean of two independent experiments. For (E), strains used were rad51د (SKY6218),

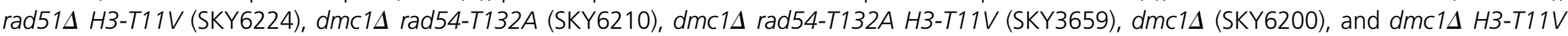
(SKY6220). (F) Spore viabilities in plasmid shuffle strains expressing wild-type H3 or H3-T11A. Three independent clones isolated for each genotype were sporulated and tetrads were dissected in three separate experiments. Each point represents the value from a single isolate $(n=30-32$ tetrads per data point). See Table 1 for summary and text for statistical test. Strains used were H3 wild type (SKY3438-3440) and H3-T11A (SKY3441-3443). (G) Evidence that the H3-T11V mutation increases MI nondisjunction in a rad54-T132A dmc1A background. The distribution of viable spores in tetrads is shown for the indicated strains. An increase in two- and zero-spore-viable (rather than three- or one-spore-viable) tetrads suggests an increased frequency of MI nondisjunction. Strains were dmc1s rad54-T132A (SKY6210) and dmc1s rad54-T132A H3-T11V (SKY3659). hht2- $\Delta$ N, H3 lacking its N-terminal 30 amino acids; n.d., not determined. 
Table 1 Absence of H3 T11ph does not compromise spore viability

\begin{tabular}{|c|c|c|c|}
\hline Mutation methoda & $H 3$ genotype $^{b}$ & Strain & Spore viability in $\%$ (no. of tetrads) \\
\hline \multirow[t]{2}{*}{ Replacement } & Wild type & 2701 & $97(44)$ \\
\hline & S10A T11V & 2705 & $97(44)$ \\
\hline \multirow[t]{8}{*}{ H3-H4 integration } & Wild type & 3311 & $96(22)$ \\
\hline & S10A & 3333 & $97(64)$ \\
\hline & $T 11 \mathrm{~V}$ & 3342 & $95(64)$ \\
\hline & S10A T11V & 3334 & $93(64)$ \\
\hline & $T 11 A$ & 3312 & $95(86)$ \\
\hline & T11S & 3313 & $99(22)$ \\
\hline & $T 11 D$ & 3332 & $97(64)$ \\
\hline & T11E & 3303 & $97(64)$ \\
\hline \multirow[t]{3}{*}{ Four-core integration } & Wild type & 3330 & $94(22)$ \\
\hline & $T 11 \mathrm{~V}$ & 3264 & $97(64)$ \\
\hline & $\Delta N$ & 2388 & $81(44)$ \\
\hline \multirow[t]{2}{*}{ Plasmid shuffle, experiment $1^{c}$} & Wild type & $3438-3440$ & $90(90)$ \\
\hline & T11A & $3441-3443$ & $86(90)$ \\
\hline \multirow[t]{2}{*}{ Plasmid shuffle, experiment $2^{c}$} & Wild type & $3438-3440$ & $75(92)$ \\
\hline & T11A & $3441-3443$ & $77(94)$ \\
\hline \multirow[t]{2}{*}{ Plasmid shuffle, experiment $3^{c}$} & Wild type & $3438-3440$ & $74(96)$ \\
\hline & T11A & $3441-3443$ & $76(96)$ \\
\hline
\end{tabular}

a Replacement: Both HHT1 and HHT2 were replaced with wild-type or mutant copies at their endogenous locations. Integration: Stable integration at leu2::hisG of a cassette carrying either the $\mathrm{H} 3$ and $H 4$ gene pair HHT2-HHF2, or all four core histone genes HTA1-HTB1 and HHT2-HHF2. Endogenous loci (encoding H3 and H4 or all four core histones, respectively) were deleted. Plasmid shuffle: Replacement of a URA3 plasmid carrying wild-type HHT2-HHF2 with a LEU2 plasmid carrying either wild-type or mutant versions. The endogenous $\mathrm{H3}$ and $\mathrm{H} 4$ loci were deleted.

${ }^{b}$ Genotypes are homozygous unless plasmid based. See Table $\mathrm{S} 1$ for complete genotypes.

${ }^{C}$ Three independent 5-FOA ${ }^{R}$ colonies were isolated for each shuffle plasmid and were dissected separately. The dissections were performed on three separate occasions by two different investigators; all six strains were dissected in parallel in each experiment. A breakdown of results by strain and experiment is provided in Figure 4F. Neither $\mathrm{H3}$ genotype nor clone identity was a significant predictor of altered spore viability $(P \geq 0.9$, linear regression).

similar to full-length $\mathrm{H} 3$ in vegetative cells (Figure 4A, lanes 23-24). This mutant displayed vegetative growth defects (Figure 4B), delayed and less efficient meiotic divisions (Figure 4D), and reduced spore viability (Table $1 ; P=0.0047$, Fisher's exact test).

\section{H3 T11ph contributes weakly to Mek1 function in the absence of Rad54 T132 phosphorylation}

Because H3-T11 mutations caused no overt defects on their own, we asked whether H3 T11ph might be redundant with Mek1 phosphorylation of Rad54 on T132 (Niu et al. 2009). A rad54-T132A mutation has little effect by itself, but in a $d m c 1 \Delta$ background it allows enough Rad51 activity to partially bypass arrest and produce some viable spores (Niu et al. 2009).

In a rad54-T132A dmc1s background, H3-T11V mutation significantly reduced spore viability (Table $2 ; P=0.0087$, Fisher's exact test), with a decrease in four-spore-viable tetrads and an increase in two- and zero-spore-viable tetrads (Figure 4G). This segregation pattern suggests increased MI nondisjunction, as expected when intersister recombination is mediated by Rad51. In this context, $H 3-T 11 \mathrm{~V}$ gave no increase in overall meiotic division efficiency (Figure 4E).

These results suggest that $\mathrm{H} 3 \mathrm{~T} 11$ phosphorylation provides a modest contribution to Mek1 function when meiotic recombination defects are encountered. Possible roles of $\mathrm{H} 3$ $\mathrm{T} 11 \mathrm{ph}$ in these contexts are addressed in the Discussion. However, since the H3-T11 mutation by itself does not detectably phenocopy a mek $1 \Delta$ mutant, we conclude that $\mathrm{H} 3 \mathrm{~T} 11 \mathrm{ph}$ is normally dispensable for Mek1 function.

\section{H3 T11ph is enriched at axis-associated sites and, less strongly, along chromatin loops}

H3 T11ph has been used as a cytological marker for Mek1 activity (Subramanian et al. 2016). Given our results establishing that $\mathrm{H} 3$ is a direct phosphorylation target of Mek1, we reasoned that $\mathrm{H} 3 \mathrm{~T} 11 \mathrm{ph}$ would also provide a sensitive and specific marker to reveal the genomic locations of active Mek1 kinase. We therefore assessed genome-wide H3 T11ph by ChIP-seq.

Samples were collected at 3 and $4 \mathrm{hr}$ in meiosis from each of two independent wild-type cultures. To control for specificity of the H3 T11ph ChIP-seq signal, a sample was also collected from a 3.5-hr culture of a spo11-Y135F mutant. A set amount of $S$. pombe meiotic cells ( $4.5 \mathrm{hr}$ in meiosis; $1 \%$ of the number of $S$. cerevisiae cells) was added to each $S$. cerevisiae cell sample prior to extract preparation. Mononucleosomes were liberated from formaldehyde-fixed meiotic chromatin by digestion with MNase, immunoprecipitated with the polyclonal anti-H3 or monoclonal anti-H3 T11ph antibodies, the DNA was then purified, deep sequenced, and reads were mapped to the $S$. cerevisiae and $S$. pombe genomes. Each $S$. cerevisiae coverage map was normalized according to the $S$. pombe read density for the same antigen from the same culture (Figure 5, A-C, and Figure S2, A and B). The S. pombe spike-in control served two purposes. First, it helped minimize the effects of sample-to-sample variation in lysis, immunoprecipitation, and sequencing library preparation. Second, because the ratio of $S$. cerevisiae to $S$. pombe cells was fixed, the spike-in control provided a scaling factor to compare the relative yield of $\mathrm{H} 3$ or $\mathrm{H} 3 \mathrm{~T} 11$ ph between different $S$. cerevisiae 
Table 2 Combining H3 T11 mutations with other mutations

\begin{tabular}{llcc}
\hline $\begin{array}{l}\text { Additional } \\
\text { mutation(s) }\end{array}$ & H3 genotype & Strain & $\begin{array}{c}\text { Spore viability in } \\
\% \text { (no. of tetrads) }\end{array}$ \\
\hline H2A-S129A & Wild type & 3265 & $97(64)$ \\
T11V & 3331 & $95(64)$ \\
set1A & Wild type & 4415 & $97(42)$ \\
rad51A & S10A T11V & 3329 & $97(64)$ \\
rad54-T132A dmc1D & Wild type & 3183 & $0(44)$ \\
& S10A T11V & 3186 & $0(44)$ \\
& Wild type & 6210 & $51(80)$ \\
\hline
\end{tabular}

${ }^{a}$ Genotypes are homozygous. See Table S1 for complete genotypes.

samples (Figure 5, A-C). Note that this allows comparison between samples for the same antigen, but does not quantify the yields of different antigens relative to one another. This approach was developed independently, but is similar to the previously described use of Candida glabrata as a spike-in control to calibrate $S$. cerevisiae ChIP-seq experiments (Hu et al. 2015).

Several lines of evidence establish that these maps reported the distribution of H3 and DSB-dependent H3 T11ph with good specificity. At fine scale, H3 ChIP-seq coverage was low in promoters and showed prominent nucleosome-width peaks in coding sequences (Figure 5C), as expected for promoterassociated nucleosome-depleted regions (NDRs), and positioned nucleosomes in gene bodies (Jiang and Pugh 2009). Replicate samples agreed well, with all five H3 ChIP samples showing highly correlated distributions whether considered genome wide (Figure 5D) or at individual loci (Figure S2C). For H3 T11ph, the four wild-type maps correlated well with one another but correlated poorly with either H3 ChIP-seq or H3 T11ph from spo11-Y135F (Figure 5D), as expected if the ChIP-seq signal was specific for this Mek1-dependent histone modification. Moreover, relative to the $S$. pombe spike-in, S. cerevisiae H3 ChIP-seq coverage was similar in wild-type and spo11-Y135F samples (Figure S2C), but H3 T11ph ChIPseq coverage was substantially higher in all four wild-type samples than in spo11-Y135F (range of 3.4- to 11.6-fold across samples for genome-wide average) (Figure 5, A-C, and Figure S2D). The magnitude of the $\mathrm{H} 3 \mathrm{~T} 11 \mathrm{ph}$ signal (relative to spike-in) differed by up to $\sim 3.6$-fold between the wild-type samples, possibly due to differences in read depth, culture synchrony, or efficiency. Nevertheless, the spatial patterns were highly reproducible (Figure S2D), so maps for wild type were averaged for further analysis.

At fine scale, H3 T11ph ChIP coverage showed depletion in NDRs and nucleosomal peaks at similar positions as in the $\mathrm{H} 3$ map (Figure 5C). This pattern is as expected since presence of a nucleosome (as revealed by bulk $\mathrm{H} 3$ localization) is a prerequisite for placement of $\mathrm{H} 3 \mathrm{~T} 11 \mathrm{ph}$ by Mek1. However, when maps were examined at larger size scales, H3 T11ph showed broad hills and valleys that were not matched in the H3 ChIP-seq (Figure 5, A and B), revealing that H3 T11ph tends to be relatively enriched or depleted in domains several kilobase in width.
A priori, we envisioned two nonexclusive scenarios that might describe H3 T11ph localization: Enrichment at chromosome axes because that is where Mek1 protein is enriched cytologically and Mek1 interacts with axis proteins (Bailis and Roeder 1998; Wan et al. 2004; Carballo et al. 2008), or enrichment centered on DSB hotspots because Mek1 activation is a response to DSBs and Mek1 regulates DSB repair. We examined each possibility in turn.

Axis-associated sites: To test if H3 T11ph is enriched near axes, we compared its ChIP-seq signal with the genome-wide distribution of axis component Red1. For this purpose, we used new ChIP-seq data acquired at $3 \mathrm{hr}$ in meiosis from a strain carrying FLAG-tagged Red1 (Figure 5, A-C) as well as earlier tiling microarray-based ChIP data (ChIP-chip) (Panizza et al. 2011). Spatial patterns for both ChIP methods agreed well (Figure S3, A and B). The sites where ChIP signals for Red 1 and other axis proteins are enriched are generally assumed to be the chromatin loop bases that are embedded in the chromosome axis (Blat et al. 2002; Panizza et al. 2011; Sun et al. 2015). These sites often, but not always, overlap with intergenic regions between convergent transcription units, presumably because transcription can push cohesin and associated axis proteins along chromosomes (Lengronne et al. 2004; Bausch et al. 2007; Sun et al. 2015).

Across individual chromosomal segments, peaks and valleys in the H3 T11ph signal appeared to correspond with peaks and valleys of Red1 (Figure 5A). Confirming this impression, average H3 T11ph signal formed a broad peak $\sim 4 \mathrm{~kb}$ wide when centered on Red1 ChIP-seq peaks, slightly wider than the average of Red1 itself (Figure 5E). No such enrichment was observed in the spo11-Y135F mutant (Figure 5, A and E). H3 ChIP-seq showed no enrichment centered on Red1 peaks and was indistinguishable in wild type and spo11-Y135F (Figure 5E). Thus, H3 T11ph signal remained elevated at Red1 peak positions after correcting for bulk H3 levels (green line in Figure 5E). Furthermore, H3 T11ph ChIP-seq correlated well genome wide with Red1 ChIP-seq, whereas H3 ChIP-seq correlated poorly with Red1 (Figure 5F). Similar results were obtained by comparing H3T11ph signal to ChIP-chip data for Red1 and another axis component, Hop1 (Figure S3, C and D). We conclude that $\mathrm{H} 3 \mathrm{~T} 11 \mathrm{ph}$ is particularly prevalent where Red 1 and Hop 1 are enriched, and thus that Mek1 is highly active at axis-associated sites.

Around DSB hotspots: To test if $\mathrm{H} 3 \mathrm{~T} 11 \mathrm{ph}$ is enriched near DSB sites, we compared its ChIP-seq signal with DSB maps generated by sequencing of Spo11 oligos (Pan et al. 2011; Mohibullah and Keeney 2017). When centered on Spo11oligo hotspots, histone ChIP-seq coverage showed a complex pattern of highly localized enrichment and depletion (Figure $5 G)$. The average for total histone H3 showed strong depletion in hotspot centers, flanked by shallow alternating peaks and valleys (gray line in Figure 5G). This is the expected pattern from prior studies, reflecting the strong preference 
A
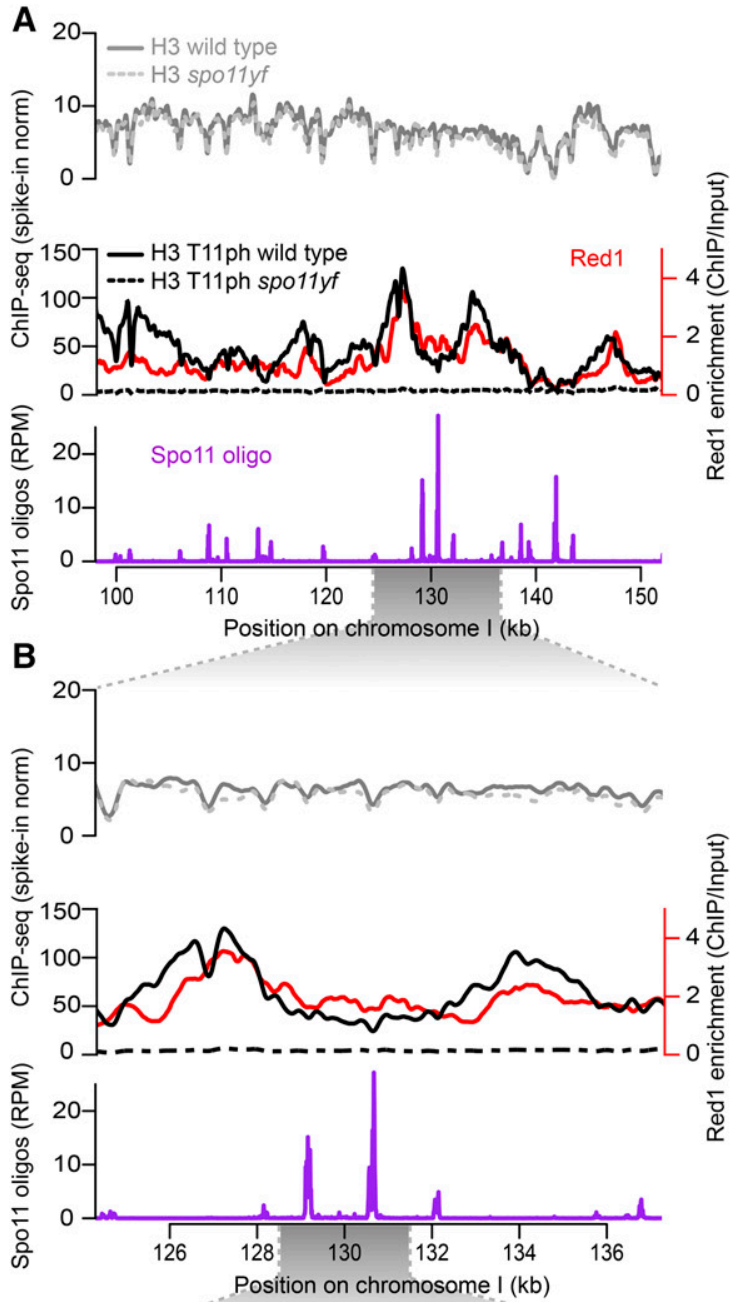

C

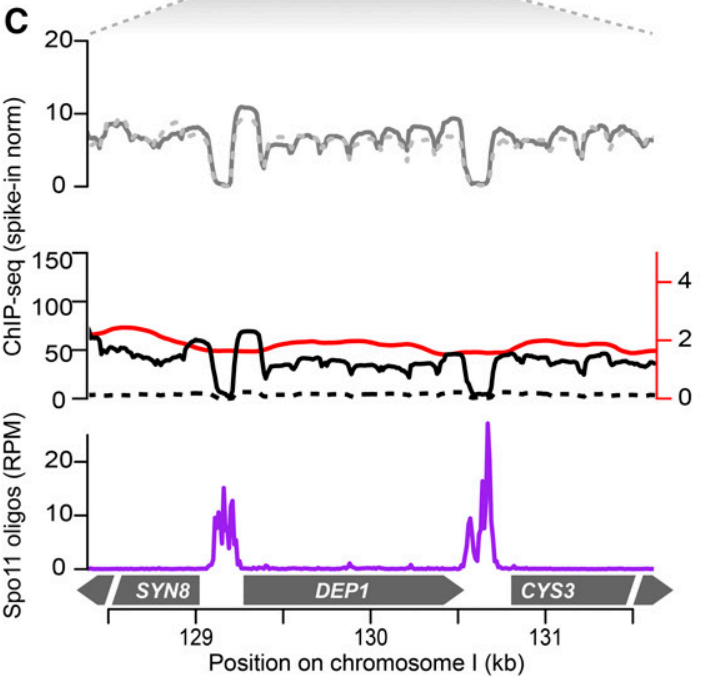

D

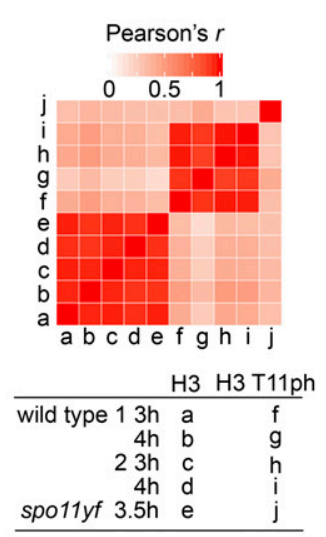

E
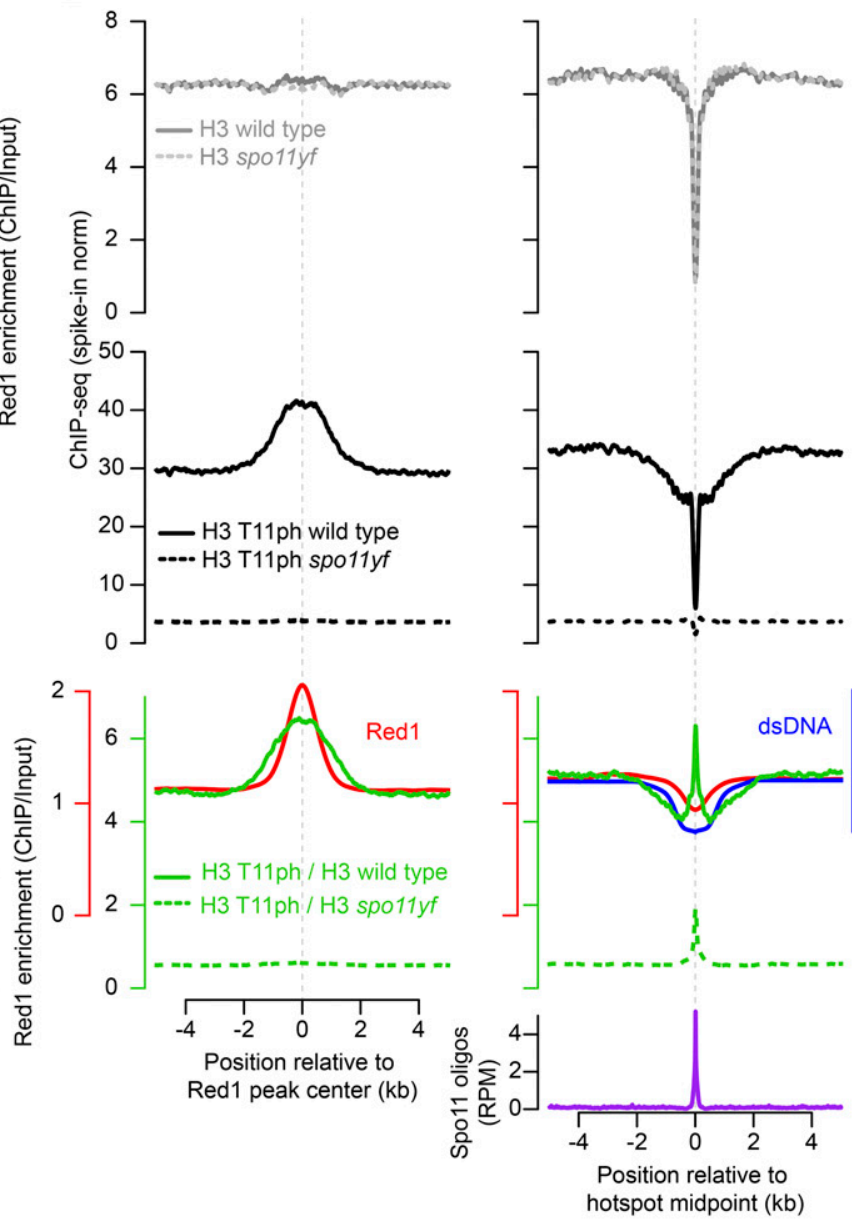

$\mathbf{F}$

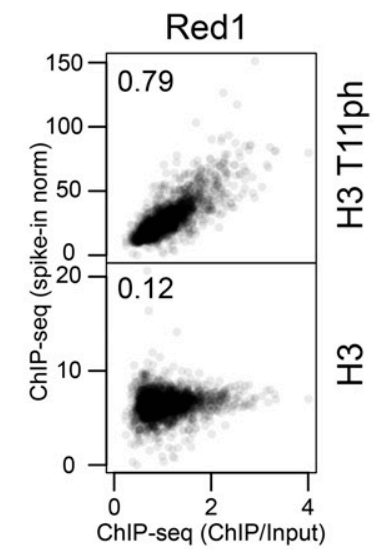

G
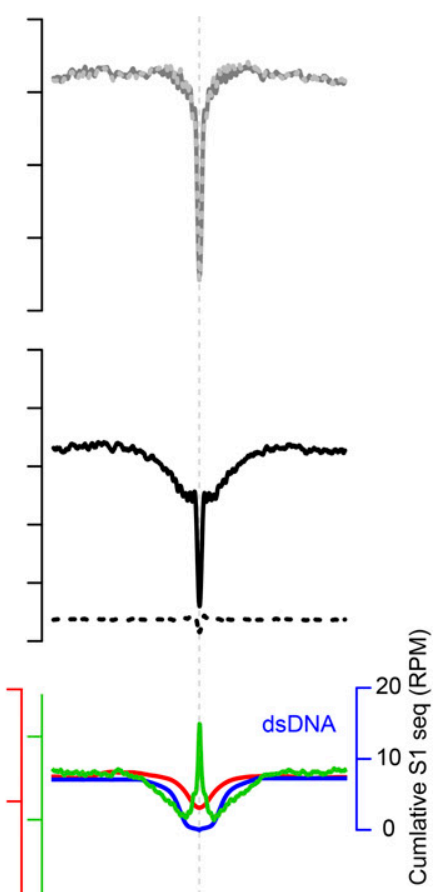

Figure 5 Spatial disposition of H3 T11ph along meiotic chromosomes. (A-C) Anti-H3 and anti-H3 T11ph ChIP-seq coverage across representative genomic regions. Coverage data for each chromosome were normalized (norm) relative to an S. pombe spike-in control. The four wild-type samples were averaged; the single spo11-Y135F sample (denoted spo11yf) is presented separately. For a given antigen (H3 or H3 T11ph), use of the internal S. pombe control allows direct quantitative comparison of relative yield in different samples, revealing in particular the DSB-dependent H3 T11ph signal via comparison of wild type with spo11-Y135F. The Spo11-oligo map [reads per million (RPM)] (Mohibullah and Keeney 2017) and anti-Red1 ChIP-seq data (this study) are shown for comparison. The H3 and H3 T11ph data shown in (A and B) and all Red1 data were smoothed with 500-bp Parzen (triangular) sliding window. Color coding is retained in the other panels in this figure. (D) Reproducibility of histone ChIP-seg coverage maps. ChIP-seq coverage was averaged in $500 \mathrm{bp}$ windows and compared between data sets. The heat map is shaded according to the Pearson's $r$ value for each 
for DSBs in $S$. cerevisiae to form in promoter NDRs that are flanked by positioned nucleosomes (Ohta et al. 1994; Wu and Lichten 1994; Pan et al. 2011) (e.g., Figure 5C). [For clarity, the plots show averages for the hottest $25 \%$ of all hotspots after excluding unusually wide hotspots (>500 bp); qualitatively similar results were obtained if all hotspots were averaged (data not shown).]

The average H3 T11ph ChIP-seq signal differed from this pattern in informative ways (black line in Figure 5G). First, at all positions across the averaging window, H3 T11ph ChIPseq signal was much higher in wild type than in spo11-Y135F, and this difference was greater for stronger hotspots than for weaker ones (Figure 5G). Therefore, there is substantial DSBdependent (thus presumably Mek1-dependent) H3 T11 phosphorylation all across the regions where DSBs usually form, not just at nearby axis sites.

Second, relative to the baseline genomic H3 T11ph signal, there was strong depletion at hotspot centers, indicated by the narrow cleft ( $\sim 200$ bp wide) in the average profile (Figure $5 \mathrm{G})$. This cleft corresponded well to the central cleft in the $\mathrm{H} 3$ ChIP-seq average, so we infer that this narrow zone of depletion reflects the fact that there are few histones available to be phosphorylated within the NDRs where hotspots generally occur. There was a peak at hotspot centers when the H3 T11ph levels were normalized to bulk H3 signal (green line in Figure 5G). However, there was also a peak when the spo11Y135F map was normalized for bulk H3, thus much or all of this is a DSB-independent signal. This may be a ChIP-seq artifact, or could reflect a low level of Mek1-independent H3 T11ph enriched near promoters (Li et al. 2015). [Although gene promoters have lower nucleosome occupancy compared with the rest of the genome, they are not devoid of nucleosomes. For example, some promoters contain positioned, high-occupancy nucleosomes; some contain nucleosomes but only in a fraction of the population; and some contain subnucleosomal histone particles (Jiang and Pugh 2009; Floer et al. 2010; Weiner et al. 2010).]

Third, there was a broader zone of lower H3 T11ph signal flanking the central NDR and extending $\sim 2 \mathrm{~kb}$ on either side (Figure 5G). This zone extended into areas where bulk H3 levels were high, so the difference map (normalizing H3 $\mathrm{T} 11 \mathrm{ph}$ to $\mathrm{H} 3$ ) revealed depletion for $\mathrm{H} 3 \mathrm{~T} 11 \mathrm{ph}$ relative to immediate surroundings (green line in Figure 5G). Nonetheless, the H3 T11ph signal across this region was substantially higher in wild type than in spo11-Y135F. Much of this depleted zone corresponded to the same areas degraded by exonucleolytic resection, as measured by S1-seq (Mimitou et al. 2017) (blue line in Figure 5G). This suggests that some or all of this depletion reflects disruption of chromatin, and thus of ChIP-detectable H3 T11ph signal, accompanying DSB resection. Interestingly, the $\mathrm{H} 3 \mathrm{~T} 11 \mathrm{ph}$ depletion zone correlated with the dimensions of a zone that was also relatively depleted for Red 1 at $3 \mathrm{hr}$ in the ChIP-seq data (Figure 5G) and in the ChIP-chip data (Figure S3E).

Collectively, these findings suggest that the distribution of DSB-provoked H3 T11 phosphorylation is governed largely by the distribution of Red1 and other proteins that are directly involved in Mek1 activation. Further implications are addressed in the Discussion.

\section{H3 T11ph correlates with DSB frequency across large subchromosomal domains}

We next examined larger scale variation in H3 T11ph ChIP signal across chromosomes. H3 T11ph ChIP signals were binned in nonoverlapping windows of varying sizes from 0.5 to $40 \mathrm{~kb}$ and then compared (Pearson's $r$ ) to Spo11-oligo densities or ChIP signals for Red1, Hop1, or Rec8 in the same bins (Figure 6). Comparisons using the ratio of H3 T11ph to H3 show which correlations are specific for the histone modification ChIP per se (green points in Figure 6) as opposed to underlying (background) enrichment or depletion in the bulk chromatin map (total H3; gray points). Comparisons using the ratio of wild type to spo11-Y135F for H3 T11ph show which correlations are specific for DSB-dependent (and thus Mek1-dependent) signal (blue points in Figure 6).

For small windows $(<2 \mathrm{~kb})$, both $\mathrm{H} 3$ and $\mathrm{H} 3 \mathrm{~T} 11 \mathrm{ph}$ were anticorrelated with Spo11-oligo density (Figure 6A). This pattern is driven by strong preference for DSBs to form in NDRs, and the attendant depletion of histone signal around hotspots (Figure 5G). In contrast, with large windows, the H3 T11ph signal instead had a significant positive correlation with Spo11-oligo density, with Pearson's $r$ values high over a range of $\sim 25-40 \mathrm{~kb}$ (Figure $6 \mathrm{~A}$ ). This correlation was also high when the wild-type H3 T11ph ChIP data were normalized to coverage in spo11-Y135F, but no such correlation was seen for total histone $\mathrm{H} 3$, thus this pattern is specific for DSBdependent H3 T11 phosphorylation. We infer that subchromosomal domains $10 \mathrm{~s}$ of $\mathrm{kb}$ wide that experience more DSBs also incur more Mek1 activity on average. This finding fits with the expectation that $\mathrm{H} 3 \mathrm{~T} 11 \mathrm{ph}$ is a faithful molecular reporter of DSB-provoked Mek1 kinase activity.

In contrast to the wide variation in correlation behavior depending on window size when $\mathrm{H} 3 \mathrm{~T} 11$ ph was compared to Spo11-oligo density, comparisons with either Red1 or Hop1

pairwise comparison. (E) H3 T11 ph enrichment around presumed axis attachment sites. H3 (top graph) and H3 T11 ph ChIP-seq coverage (middle graph) and smoothed (500-bp Parzen window) Red1 ChIP-seq data were averaged $\sim 1717$ Red1 ChIP-seq peaks. The green lines in the bottom graph show the ratio of H3 T11 ph to H3 ChIP-seq. (F) H3 T11 ph correlates well with Red1 genome wide. Each point compares the H3 or H3 T11ph ChIP-seq coverage in wild type with Red1 ChIP-seq signal averaged across nonoverlapping 5-kb bins. Correlation coefficients (Pearson's $r$ ) are indicated in each plot. (G) H3 T11 ph around DSB hotspots. ChIP-seq and Spo11-oligo data were averaged around Spo11-oligo hotspots and plotted as in (E) (Mohibullah and Keeney 2017); for clarity, hotspots $>500$ bp wide were excluded, and only the hottest $25 \%$ of hotspots are shown ( $N=872$ hotspots). Note that vertical and horizontal scales for ChIP-seq data are the same in ( $E$ and $G$ ) to facilitate direct comparison. The blue line in the third panel shows the extent of doublestranded DNA depletion predicted from S1-seq mapping of DSB resection tracts around the same hotspots (Mimitou et al. 2017). The lowest panel shows the average Spo11-oligo profile. 


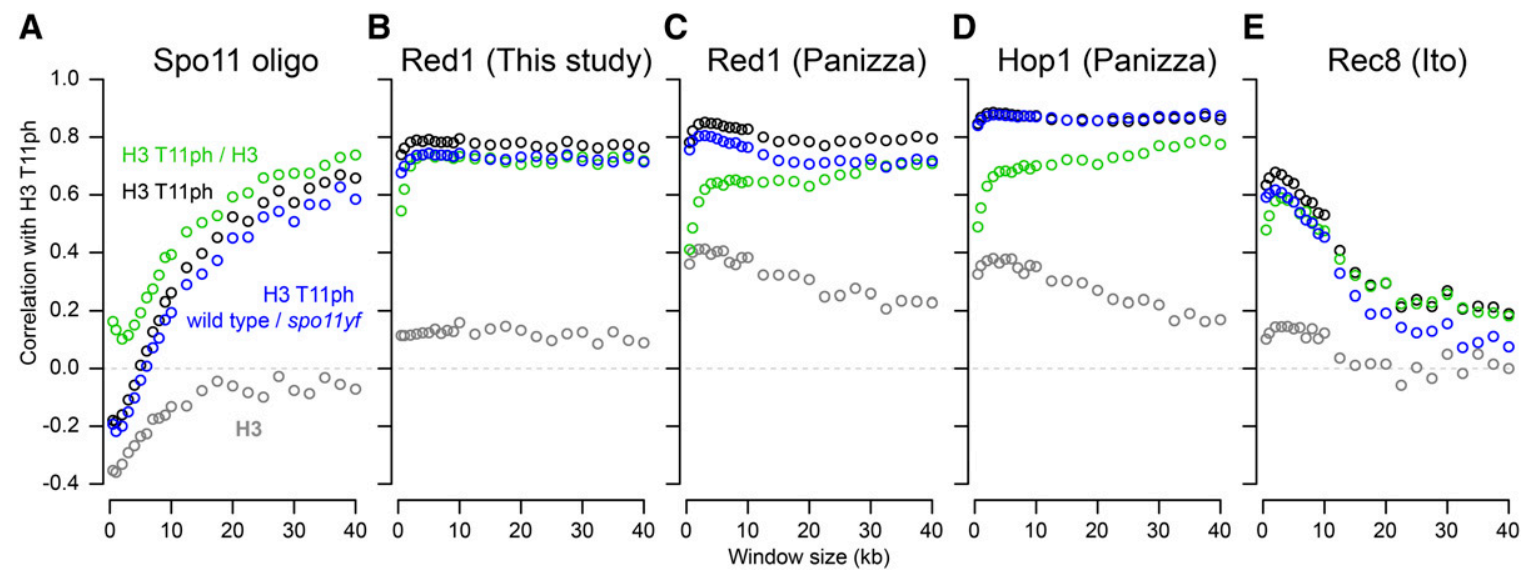

Figure 6 Scale-dependent correlations of H3 T11ph with chromosomal features. Anti-H3 (gray points) and anti-H3 T11ph (black points) ChIP-seq coverage was binned in nonoverlapping windows of varying sizes and compared (Pearson's $r$ ) to Spo11-oligo density (A) or ChIP-chip or ChIP-seq signals for Red1 (B and C), Hop1 (D), or Rec8 (E) averaged across the same windows. Red1 ChIP-seq data are from this study. Spo11-oligo (Mohibullah and Keeney 2017), Red1 and Hop1 ChIP-chip (Panizza et al. 2011), and Rec8 ChIP-seq data (Ito et al. 2014) were from previous studies. Green points show correlations using the ratio of H3 T11ph to H3 in wild type; blue points show correlations for the ratio of wild type to spo11-Y135F H3 T11ph signal.

ChIP showed strong positive correlations over all window sizes tested (Figure 6, B-D). Qualitatively similar results were obtained with Red1 ChIP-seq and ChIP-chip data, the principal difference being that the Red1 ChIP-chip data showed a higher but still weak correlation with bulk histone H3 ChIP for smaller window sizes (compare Figure 6, B and C). Higher resolution and better specificity of sequencing $v s$. hybridizationbased detection may explain this subtle difference between the ChIP-seq and ChIP-chip data for Red1. The good concordance of the ChIP-seq and ChIP-chip data for Red1 validates the use of published Hop1 ChIP-chip data. Hop1 was highly similar to Red1 in showing a largely scale-independent positive correlation with H3T11ph (Figure 6, C and D).

Using Rec8 ChIP-seq data previously collected at $4 \mathrm{hr}$ (Ito et al. 2014), H3 T11ph showed a positive correlation for short windows (less than $\sim 10 \mathrm{~kb}$ ) but only weak correlation with larger windows (Figure 6E). This pattern can be understood as the combination of two spatial correlations with different length dependencies. At short distances $(<10 \mathrm{~kb})$, Mek1 activity is particularly enriched at preferred binding sites for Red1, Hop1, and Rec8 (i.e., presumptive axis attachment sites; Figure 5E). At longer distances (10s of kb), the domains that are relatively DSB rich (and thus have more Mek1 activity) are also enriched for Red1 and Hop1 but not for Rec8 (Blat et al. 2002; Pan et al. 2011; Panizza et al. 2011; Ito et al. 2014).

\section{Discussion}

This study and others (Govin et al. 2010; Subramanian et al. 2016) establish that H3 T11 phosphorylation is highly induced during meiosis in S. cerevisiae. Our findings additionally demonstrate that $\mathrm{H} 3 \mathrm{~T} 11 \mathrm{ph}$ is a direct product of DSBinduced activation of Mek1. Mek1 is conserved in S. pombe (Perez-Hidalgo et al. 2003), so it seems likely that this kinase is also responsible for the $\mathrm{H} 3 \mathrm{~T} 11 \mathrm{ph}$ we observed in fission yeast.
Mek1 appears specifically in fungal taxa, but the larger Rad53 kinase family is ubiquitous in eukaryotes (Subramanian and Hochwagen 2014). Another member of this family, CHK1, was reported to be required for $\mathrm{H} 3 \mathrm{~T} 11 \mathrm{ph}$ in mouse fibroblasts (Shimada et al. 2008). In this case, however, DNA damage caused a decrease in $\mathrm{H} 3 \mathrm{~T} 11 \mathrm{ph}$ levels. It remains unknown if CHK1 directly phosphorylates $\mathrm{H} 3 \mathrm{~T} 11$ or if $\mathrm{H} 3 \mathrm{~T} 11 \mathrm{ph}$ occurs in response to DSBs in mammalian meiosis. $\mathrm{H} 3 \mathrm{~T} 11$ ph has been reported during meiosis in sciarid flies (Escriba et al. 2011), suggesting evolutionary conservation beyond yeasts.

H3 T11 can also be directly phosphorylated by pyruvate kinase M2 in S. cerevisiae and mammalian cells, possibly to coordinate chromatin structure and gene expression with the cell's nutritional status (Yang et al. 2012; Li et al. 2015). In cultured human cells, H3 T11ph is also formed by protein kinase C-related kinase 1 near promoters of androgen receptor-modulated genes (Metzger et al. 2008), and by deathassociated protein-like kinase during mitosis, particularly near centromeres (Preuss et al. 2003). Our results establish that meiotic induction of $\mathrm{H} 3 \mathrm{~T} 11 \mathrm{ph}$ in yeasts is fundamentally distinct from these other modes of H3 T11 phosphorylation in terms of provenance and genomic distribution.

\section{Possible functions of $\mathrm{H3}$ T11ph in meiosis}

Under the conditions in this study, histone mutations that eliminated H3 T11 phosphorylation caused no discernible meiotic defects by themselves. This was true with multiple independent mutagenesis strategies and numerous mutant constructs encoding different amino acid substitutions alone or in combination with mutation of $\mathrm{H} 3 \mathrm{~S} 10$. We conclude that $\mathrm{H} 3 \mathrm{~T} 11 \mathrm{ph}$ is dispensable for meiosis under our standard conditions.

Why our results differed from a previous report (Govin et al. 2010) remains unknown. One possibility is that the highly variable spore viability in the plasmid shuffle system fortuitously gave the incorrect appearance of a meiotic defect 
in the earlier study. The reported decrease in spore viability [from $\sim 80 \%$ in the control to $\sim 50 \%$ with $H 3-T 11 A$ (Govin et al. 2010)] was of comparable magnitude to the intrinsic experimental variability we observed with plasmid-borne histone cassettes. Alternatively, studies in the two laboratories may have had undocumented differences in sporulation conditions to which H3-T11 mutants are specifically sensitive.

Despite H3 T11ph being dispensable in unperturbed meiosis, we did observe that the H3-T11V mutation modestly exacerbated the phenotype of a $d m c 1 \Delta$ rad54-T132A mutant. One possibility is that $\mathrm{H} 3-\mathrm{T} 11 \mathrm{~V}$ protein acts as a weak competitive inhibitor of Mek1, thereby attenuating its ability to phosphorylate other substrates. However, we favor the alternative that the effect of the H3- $111 \mathrm{~V}$ mutation is attributable to absence of H3 T11 phosphorylation per se. Possibly, H3 T11ph helps Mek1 maintain residual interhomolog bias when Rad51 is the sole source of strand exchange activity. In this model, increased MI nondisjunction is caused by more of the residual DSB repair being between sister chromatids, and less between homologs. This interpretation is motivated by the increased intersister recombination observed in a rad54T132A mutant when Mek1 activity is inhibited, and by the ability of the rad54-T132A mutation to rescue some spore viability in a $d m c 1 \Delta$ background but not in $d m c 1 \Delta$ mek1s (Niu et al. 2009). These findings indicate that other Mek1 targets contribute to interhomolog recombination by Rad51 when Dmc1 is missing and Rad54 cannot be phosphorylated. The recent discovery that Mek1 phosphorylates Hed1 and histone H2B make these strong candidates for additional redundancy (Callender et al. 2016; Suhandynata et al. 2016) (N. M. Hollingsworth and H. Niu, unpublished data).

If $\mathrm{H} 3 \mathrm{~T} 11 \mathrm{ph}$ does promote Mek1 function, albeit in a minor way, what might its role be? One possibility is that it is an effector of Mek1 signaling. This could be via recruitment to chromatin of proteins with phospho-threonine binding motifs such as the FHA domain, which is present in numerous proteins in $S$. cerevisiae including the recombination protein Xrs2 (Mahajan et al. 2008; Matsuzaki et al. 2008). Or, H3 T11ph might impinge on nucleosome stability; higher order chromatin organization; or ability to install, remove, or read other histone modifications. We observed potential cross-talk between histone modifications in that $\mathrm{H} 3 \mathrm{~S} 10$ ph blocked the ability of Mek1 to phosphorylate T11 on the same peptide. Cross-talk of H3 T11ph with other H3 modifications has been documented in vegetatively growing yeast [H3 K4 methylation (Li et al. 2015)] and in human cells [H3 K9 acetylation (Yang et al. 2012) and demethylation (Metzger et al. 2008)]. A second, nonexclusive possibility is that $\mathrm{H} 3 \mathrm{~T} 11 \mathrm{ph}$ might maintain or amplify Mek1 activity via positive feedback. For example, the FHA domain of Mek1 might bind directly to H3 $\mathrm{T} 11 \mathrm{ph}$ in a manner that stabilizes or increases the amount of active Mek1. Both general types of role, downstream effector or feedback amplifier, are compatible with the observed genetic interaction of H3-T11 mutation with $d m c 1 \Delta$ rad54-T132A.

\section{Spatial organization of Mek1 activity}

Although H3 T11 can apparently be phosphorylated by other kinases, the magnitude of the DSB- and Mek1-dependent signal combined with its rapid disappearance when Mek1 is shut off made H3 T11ph an excellent candidate for a molecular marker of ongoing Mek1 activity. Our experiments establish proof of principle for this use in genomic experiments, and also validate that cytological staining for $\mathrm{H} 3 \mathrm{~T} 11 \mathrm{ph}$ provides a direct readout of Mek1 activity (Subramanian et al. 2016).

The most prominent sites of $\mathrm{H} 3 \mathrm{~T} 11 \mathrm{ph}$, and thus of Mek1 activity, were coincident with peaks of Red1 and Hop1, i.e., presumed axis attachment sites. This pattern is not surprising given that Mek1 protein appears to be enriched on axes as assessed by immunocytology (Bailis and Roeder 1998). However, immunolocalization does not reveal kinase activity per se, and cannot evaluate the degree to which activity might spread in cis. Interestingly, the H3 T11ph enrichment was similar to that of Red1 and Hop1 around axis sites. This more constrained pattern contrasts with the broad, relatively unstructured spread of $\gamma-\mathrm{H} 2 \mathrm{~A}$ over $10 \mathrm{~s}$ of $\mathrm{kb}$ around DSBs in yeast (Shroff et al. 2004). The local coincidence between enrichment patterns for $\mathrm{H} 3 \mathrm{~T} 11 \mathrm{ph}$ with those for Red1 and Hop1 could be because Mek1 protein is constrained, i.e., it rarely diffuses away from the sites where it has been activated. Alternatively, Mek1 may be rapidly inactivated if it diffuses away and/or the phosphates that Mek1 places outside the immediate vicinity of Mek1 activation sites might be more rapidly removed by phosphatases. In contrast, the broader $\gamma$-H2A distribution suggests that Mec1 and/or Tel1 activity can spread relatively uniformly around a DSB. We note that our analysis does not allow us to definitively evaluate how far H3 T11ph spreads around any given DSB, because we are looking at a population average across cells with DSBs at many different positions. It is conceivable that $\mathrm{H} 3$ T11ph (and thus Mek1 activity) spreads as far as Mec1/Tel1 activity does, given the strong correlation of $\mathrm{H} 3 \mathrm{~T} 11 \mathrm{ph}$ with Spo11-oligo density across large size scales. Rigorously addressing this question would require measuring $\mathrm{H} 3 \mathrm{~T} 11 \mathrm{ph}$ around a unitary, defined DSB.

We additionally observed substantial levels of DSBdependent H3 T11ph across areas in between Red1 peaks, i.e., along the lengths of presumptive chromatin loops. This signal correlated with Spo11-oligo frequency (i.e., local DSB density) but was lower immediately around DSB hotspots, coincident with regions also depleted of Red1 in both the ChIP-seq and ChIP-chip data sets analyzed. For any protein, ChIP enrichment at given sites does not imply that these are the only sites of binding. In yeast meiosis, axis proteins Red1 and Hop1 are also detected above background across regions in between the sites of their principal enrichment. ChIP provides a population average measurement, so possible interpretations are that Red 1 et al. are bound to different sites in different cells, but only at discrete sites in any one cell (an "axis sites only" model); or that there is lower level binding to chromatin 
loops in addition to higher level binding at axis sites (an "axis plus dispersed binding" model).

The DSB-dependent H3 T11ph ChIP signal tracked closely with Red 1 and Hop1 ChIP at all size scales, both quantitatively and spatially. Thus, our findings are consistent with Mek1 activity manifesting anywhere Red 1 and Hop1 are present to support it once DSBs have formed. It is interesting to note that the sites of highest Mek1 activity (i.e., Red1/Hop1 peaks) are spatially distinct from the DSB sites where Mek1 exerts its known biological function: Hop1/Red1-dependent control of recombination outcome. However, even though H3 T11ph ChIP signal was less abundant immediately adjacent to DSB sites than elsewhere, the DSB-proximal signal was substantially higher in wild type than in the spo11-Y135F control. Thus, we infer that active Mek1 kinase has access to chromatin and chromatin-associated proteins immediately surrounding DSBs.

A puzzle about the signal immediately adjacent to hotspots is that DSBs are exonucleolytically resected for $\sim 800 \mathrm{nt}$ on average on both sides of the break (Zakharyevich et al. 2010; Mimitou et al. 2017), but single-stranded DNA (ssDNA) should not be revealed in our ChIP-seq data even if it were still bound by histones because the sequencing library preparation protocol is not expected to support recovery of ssDNA. What then is the source of H3 T11-phosphorylated nucleosomes immediately around hotspots? Likely candidates are the sister of the broken chromatid, one or both intact chromatids of the homologous chromosome with which recombination is occurring, and/or recombination intermediates (D-loops and double Holliday junctions) if these are chromatinized. Because Mek1 controls homolog bias, we speculate that some or all of the H3 T11ph signal around hotspots is from Mek1 action on the sisters of broken chromatids. In support of this idea, we further note that the areas expected to be covered by DSB resection are also areas where the H3 T11ph signal is locally depleted. This pattern is thus in line spatially with what would be predicted if both sister chromatids are being exposed to Mek1 activity.

Our data are consistent with spreading of DSB-provoked Mek1 activity in cis along chromatin and concentrating wherever Red1 and Hop1 are also concentrated. The findings neither refute nor support the TLAC model (see Introduction), but are consistent with this model provided that DSB-dependent activation of Mek1 at axis sites can be accompanied by spreading of Mek1 activity to surrounding chromatin as well. Current versions of the TLAC model favor the idea that tethering occurs before DSB formation because some partners of Spo11 are enriched at axis sites rather than at hotspots, but can be connected to hotspots physically via interactions with a reader (Spp1) of the H3 K4 methylation that is prominent around promoters (Panizza et al. 2011; Acquaviva et al. 2013; Sommermeyer et al. 2013). Such loop-axis interactions prior to DSB formation could provide a means to rapidly and specifically activate Mek1 at a nearby axis site in response to a DSB at a hotspot within a tethered loop.

Immunostaining experiments demonstrated $\mathrm{H} 3 \mathrm{~T} 11 \mathrm{ph}$ foci of variable size and intensity on chromatin where the synap- tonemal complex had not yet formed, although spatial disposition of this phosphorylation relative to axes has not been reported (Subramanian et al. 2016). Combining these cytological findings with our genomic data suggests that each DSB provokes a relatively large zone of Mek1 activation that encompasses the broken chromatin loop, its sister chromatid, and the adjacent loop base(s). We speculate that this zone may extend across one or more loops, perhaps dependent in part on availability of sufficient amounts of Red 1 and Hop1 to support Mek1 activity.

In summary, the detection of H3 T11ph is useful as an indicator of meiotic DSB formation, an indicator of Mek1 activation level, and a marker of the spatial organization of chromatin upon which Mek1 acts. H3 T11ph ChIP will be a powerful tool for dissecting not only the function of Mek1 but also the higher order structural organization of recombining chromosomes.

\section{Acknowledgments}

We thank J. Song (Keeney laboratory) for assistance with strain construction and ChIP-seq sample preparation; K. Kugou (Ohta laboratory) for assistance with strain construction and Red1 ChIP-seq data; A. Oda (Ohta laboratory) for assistance with Red1 ChIP-seq data; F. Klein (University of Vienna) for suggesting $S$. pombe as a spike-in control for ChIP-seq; G. Smith (Fred Hutchinson Cancer Research Center) for $S$. pombe strains and advice on culturing; J. Govin and S. Berger (University of Pennsylvania) for providing histone mutant plasmids and strains and for communicating information prior to publication; C. Hughes and C. D. Allis (Rockefeller University) for providing peptides, recombinant histones, plasmids, and valuable guidance; N. Hunter (University of California, Davis) and G. Chung (Keeney laboratory) for strains; A. Viale and N. Mohibullah (Memorial Sloan Kettering Cancer Center Integrated Genomics Operation) for sequencing; and S. Yamada (Keeney laboratory) and N. Socci (Memorial Sloan Kettering Cancer Center Bioinformatics Core) for bioinformatics assistance. Memorial Sloan Kettering Cancer Center core facilities were supported by National Institutes of Health/National Cancer Institute Cancer Center support grant P30 CA-008748. R.K. was supported in part by National Institute of General Medical Sciences predoctoral training award T32 BM-008539. This work was supported by National Institutes of Health grants R01 GM-058673 and R35 GM-118092 (to S.K.) and R01 GM-050717 (to N.M.H.), and by grants from the Japan Society for the Promotion of Science (15H04625, 26291018, and $17 \mathrm{H} 03711$ to K.O.).

\section{Literature Cited}

Acquaviva, L., L. Szekvolgyi, B. Dichtl, B. S. Dichtl, C. de La Roche Saint Andre et al., 2013 The COMPASS subunit Spp1 links histone methylation to initiation of meiotic recombination. Science 339: 215-218. 
Ahn, S. H., W. L. Cheung, J. Y. Hsu, R. L. Diaz, M. M. Smith et al., 2005 Sterile 20 kinase phosphorylates histone H2B at serine 10 during hydrogen peroxide-induced apoptosis in $S$. cerevisiae. Cell 120: 25-36.

Allen, J. J., M. Li, C. S. Brinkworth, J. L. Paulson, D. Wang et al., 2007 A semisynthetic epitope for kinase substrates. Nat. Methods 4: 511-516.

Bahler, J., P. Schuchert, C. Grimm, and J. Kohli, 1991 Synchronized meiosis and recombination in fission yeast: observations with pat1114 diploid cells. Curr. Genet. 19: 445-451.

Bailis, J. M., and G. S. Roeder, 1998 Synaptonemal complex morphogenesis and sister-chromatid cohesion require Mek1-dependent phosphorylation of a meiotic chromosomal protein. Genes Dev. 12: 3551-3563.

Bailis, J. M., and G. S. Roeder, 2000 Pachytene exit controlled by reversal of Mek1-dependent phosphorylation. Cell 101: 211221.

Baudat, F., and A. Nicolas, 1997 Clustering of meiotic doublestrand breaks on yeast Chromosome III. Proc. Natl. Acad. Sci. USA 94: 5213-5218.

Bausch, C., S. Noone, J. M. Henry, K. Gaudenz, B. Sanderson et al., 2007 Transcription alters chromosomal locations of cohesin in Saccharomyces cerevisiae. Mol. Cell. Biol. 27: 8522-8532.

Bishop, D. K., D. Park, L. Xu, and N. Kleckner, 1992 DMC1: a meiosis-specific yeast homolog of $E$. coli recA required for recombination, synaptonemal complex formation, and cell cycle progression. Cell 69: 439-456.

Blat, Y., R. U. Protacio, N. Hunter, and N. Kleckner, 2002 Physical and functional interactions among basic chromosome organizational features govern early steps of meiotic chiasma formation. Cell 111: 791-802.

Borde, V., N. Robine, W. Lin, S. Bonfils, V. Géli et al., 2009 Histone H3 lysine 4 trimethylation marks meiotic recombination initiation sites. EMBO J. 28: 99-111.

Callender, T. L., R. Laureau, L. Wan, X. Chen, R. Sandhu et al., 2016 Mek1 down regulates Rad51 activity during yeast meiosis by phosphorylation of Hed1. PLoS Genet. 12: e1006226.

Carballo, J. A., A. L. Johnson, S. G. Sedgwick, and R. S. Cha, 2008 Phosphorylation of the axial element protein Hop1 by Mec1/Tel1 ensures meiotic interhomolog recombination. Cell 132: 758-770.

Cervantes, M. D., J. A. Farah, and G. R. Smith, 2000 Meiotic DNA breaks associated with recombination in $S$. pombe. Mol. Cell 5: 883-888.

Chen, X., R. T. Suhandynata, R. Sandhu, B. Rockmill, N. Mohibullah et al., 2015 Phosphorylation of the synaptonemal complex protein Zip1 regulates the crossover/noncrossover decision during yeast meiosis. PLoS Biol. 13: e1002329.

Cheng, Y. H., C. N. Chuang, H. J. Shen, F. M. Lin, and T. F. Wang, 2013 Three distinct modes of Mec1/ATR and Tel1/ATM activation illustrate differential checkpoint targeting during budding yeast early meiosis. Mol. Cell. Biol. 33: 33653376.

Chu, S., and I. Herskowitz, 1998 Gametogenesis in yeast is regulated by a transcriptional cascade dependent on Ndt80. Mol. Cell 1: 685-696.

Chuang, C. N., Y. H. Cheng, and T. F. Wang, 2012 Mek1 stabilizes Hop1-Thr318 phosphorylation to promote interhomolog recombination and checkpoint responses during yeast meiosis. Nucleic Acids Res. 40: 11416-11427.

Clark-Adams, C. D., D. Norris, M. A. Osley, J. S. Fassler, and F. Winston, 1988 Changes in histone gene dosage alter transcription in yeast. Genes Dev. 2: 150-159.

Doll, E., M. Molnar, G. Cuanoud, G. Octobre, V. Latypov et al., 2008 Cohesin and recombination proteins influence the G1to-S transition in azygotic meiosis in Schizosaccharomyces pombe. Genetics 180: 727-740.
Escriba, M. C., M. C. Giardini, and C. Goday, 2011 Histone H3 phosphorylation and non-disjunction of the maternal $\mathrm{X}$ chromosome during male meiosis in sciarid flies. J. Cell Sci. 124: 1715-1725.

Floer, M., X. Wang, V. Prabhu, G. Berrozpe, S. Narayan et al., 2010 A RSC/nucleosome complex determines chromatin architecture and facilitates activator binding. Cell 141: 407-418.

Gasior, S. L., H. Olivares, U. Ear, D. M. Hari, R. Weichselbaum et al., 2001 Assembly of RecA-like recombinases: distinct roles for mediator proteins in mitosis and meiosis. Proc. Natl. Acad. Sci. USA 98: 8411-8418.

Gerton, J. L., J. DeRisi, R. Shroff, M. Lichten, P. O. Brown et al., 2000 Inaugural article: global mapping of meiotic recombination hotspots and coldspots in the yeast Saccharomyces cerevisiae. PProc Natl Acad Sci U S A 97: 11383-11390.

Goldfarb, T., and M. Lichten, 2010 Frequent and efficient use of the sister chromatid for DNA double-strand break repair during budding yeast meiosis. PLoS Biol. 8: e1000520.

Govin, J., J. Dorsey, J. Gaucher, S. Rousseaux, S. Khochbin et al., 2010 Systematic screen reveals new functional dynamics of histones H3 and H4 during gametogenesis. Genes Dev. 24: 17721786.

Harvey, A. C., S. P. Jackson, and J. A. Downs, 2005 Saccharomyces cerevisiae histone H2A Ser122 facilitates DNA repair. Genetics 170: 543-553.

Heyer, W. D., X. Li, M. Rolfsmeier, and X. P. Zhang, 2006 Rad54: the Swiss Army knife of homologous recombination? Nucleic Acids Res. 34: 4115-4125.

Hong, S., Y. Sung, M. Yu, M. Lee, N. Kleckner et al., 2013 The logic and mechanism of homologous recombination partner choice. Mol. Cell 51: 440-453.

Hsu, J. Y., Z. W. Sun, X. Li, M. Reuben, K. Tatchell et al., 2000 Mitotic phosphorylation of histone H3 is governed by Ipl1/aurora kinase and Glc7/PP1 phosphatase in budding yeast and nematodes. Cell 102: 279-291.

Hu, B., N. Petela, A. Kurze, K. L. Chan, C. Chapard et al., 2015 Biological chromodynamics: a general method for measuring protein occupancy across the genome by calibrating ChIP-seq. Nucleic Acids Res. 43: e132.

Hunter, N., 2015 Meiotic recombination: the essence of heredity. Cold Spring Harb. Perspect. Biol. 7: a016618.

Hyppa, R. W., and G. R. Smith, 2009 Using Schizosaccharomyces pombe meiosis to analyze DNA recombination intermediates. Methods Mol. Biol. 557: 235-252.

Ito, M., K. Kugou, J. A. Fawcett, S. Mura, S. Ikeda et al., 2014 Meiotic recombination cold spots in chromosomal cohesion sites. Genes Cells 19: 359-373.

Jiang, C., and B. F. Pugh, 2009 Nucleosome positioning and gene regulation: advances through genomics. Nat. Rev. Genet. 10: 161172.

Kim, K. P., B. M. Weiner, L. Zhang, A. Jordan, J. Dekker et al., 2010 Sister cohesion and structural axis components mediate homolog bias of meiotic recombination. Cell 143: 924-937.

Kleckner, N., 2006 Chiasma formation: chromatin/axis interplay and the role(s) of the synaptonemal complex. Chromosoma 115: 175-194.

Klein, F., P. Mahr, M. Galova, S. B. Buonomo, C. Michaelis et al., 1999 A central role for cohesins in sister chromatid cohesion, formation of axial elements, and recombination during yeast meiosis. Cell 98: 91-103.

Kugou, K., T. Fukuda, S. Yamada, M. Ito, H. Sasanuma et al., 2009 Rec8 guides canonical Spo11 distribution along yeast meiotic chromosomes. Mol. Biol. Cell 20: 3064-3076.

Laemmli, U. K., 1970 Cleavage of structural proteins during the assembly of the head of bacteriophage T4. Nature 227: 680-685.

Lam, I., and S. Keeney, 2014 Mechanism and regulation of meiotic recombination initiation. Cold Spring Harb. Perspect. Biol. 7: a016634 
Lao, J. P., V. Cloud, C. C. Huang, J. Grubb, D. Thacker et al., 2013 Meiotic crossover control by concerted action of Rad51Dmc1 in homolog template bias and robust homeostatic regulation. PLoS Genet. 9: e1003978.

Leem, S. H., and H. Ogawa, 1992 The MRE4 gene encodes a novel protein kinase homologue required for meiotic recombination in Saccharomyces cerevisiae. Nucleic Acids Res. 20: 449-457.

Lengronne, A., Y. Katou, S. Mori, S. Yokobayashi, G. P. Kelly et al., 2004 Cohesin relocation from sites of chromosomal loading to places of convergent transcription. Nature 430: 573-578.

Li, H., 2013 Aligning sequence reads, clone sequences and assembly contigs with BWA-MEM. arXiv arXiv:1303.3997v2 [q-bio.GN].

Li, S., S. K. Swanson, M. Gogol, L. Florens, M. P. Washburn et al., 2015 Serine and SAM responsive complex SESAME regulates histone modification crosstalk by sensing cellular metabolism. Mol. Cell 60: 408-421.

Lo, H. C., and N. M. Hollingsworth, 2011 Using the semi-synthetic epitope system to identify direct substrates of the meiosis-specific budding yeast kinase, Mek1. Methods Mol. Biol. 745: 135-149.

Lydall, D., Y. Nikolsky, D. K. Bishop, and T. Weinert, 1996 A meiotic recombination checkpoint controlled by mitotic checkpoint genes. Nature 383: 840-843.

Mahadevaiah, S. K., J. M. Turner, F. Baudat, E. P. Rogakou, P. de Boer et al., 2001 Recombinational DNA double-strand breaks in mice precede synapsis. Nat. Genet. 27: 271-276.

Mahajan, A., C. Yuan, H. Lee, E. S. Chen, P. Y. Wu et al., 2008 Structure and function of the phosphothreonine-specific FHA domain. Sci. Signal. 1: re12.

Martini, E., R. L. Diaz, N. Hunter, and S. Keeney, 2006 Crossover homeostasis in yeast meiosis. Cell 126: 285-295.

Matsuzaki, K., A. Shinohara, and M. Shinohara, 2008 Forkheadassociated domain of yeast Xrs2, a homolog of human Nbs1, promotes nonhomologous end joining through interaction with a ligase IV partner protein, Lif1. Genetics 179: 213-225.

Meeks-Wagner, D., and L. H. Hartwell, 1986 Normal stoichiometry of histone dimer sets is necessary for high fidelity of mitotic chromosome transmission. Cell 44: 43-52.

Metzger, E., N. Yin, M. Wissmann, N. Kunowska, K. Fischer et al., 2008 Phosphorylation of histone H3 at threonine 11 establishes a novel chromatin mark for transcriptional regulation. Nat. Cell Biol. 10: 53-60.

Mimitou, E. P., S. Yamada, and S. Keeney, 2017 A global view of meiotic double-strand break end resection. Science 355: 40-45.

Mohibullah, N., and S. Keeney, 2017 Numerical and spatial patterning of yeast meiotic DNA breaks by Tel1. Genome Res. 27: 278-288.

Mok, J., P. M. Kim, H. Y. Lam, S. Piccirillo, X. Zhou et al., 2010 Deciphering protein kinase specificity through large-scale analysis of yeast phosphorylation site motifs. Sci. Signal. 3: ra12.

Murakami, H., and S. Keeney, 2014 Temporospatial coordination of meiotic DNA replication and recombination via DDK recruitment to replisomes. Cell 158: 861-873.

Murakami, H., V. Borde, A. Nicolas, and S. Keeney, 2009 Gel electrophoresis assays for analyzing DNA double-strand breaks in Saccharomyces cerevisiae at various spatial resolutions. Methods Mol. Biol. 557: 117-142.

Nady, N., J. Min, M. S. Kareta, F. Chedin, and C. H. Arrowsmith, 2008 A SPOT on the chromatin landscape? Histone peptide arrays as a tool for epigenetic research. Trends Biochem. Sci. 33: 305-313.

Niu, H., L. Wan, B. Baumgartner, D. Schaefer, J. Loidl et al., 2005 Partner choice during meiosis is regulated by Hop1-promoted dimerization of Mek1. Mol. Biol. Cell 16: 5804-5818.

Niu, H., X. Li, E. Job, C. Park, D. Moazed et al., 2007 Mek1 kinase is regulated to suppress double-strand break repair between sister chromatids during budding yeast meiosis. Mol. Cell. Biol. 27: $5456-5467$.
Niu, H., L. Wan, V. Busygina, Y. Kwon, J. A. Allen et al., 2009 Regulation of meiotic recombination via Mek1-mediated Rad54 phosphorylation. Mol. Cell 36: 393-404.

Norris, D., and M. A. Osley, 1987 The two gene pairs encoding $\mathrm{H} 2 \mathrm{~A}$ and H2B play different roles in the Saccharomyces cerevisiae life cycle. Mol. Cell. Biol. 7: 3473-3481.

Ohta, K., T. Shibata, and A. Nicolas, 1994 Changes in chromatin structure at recombination initiation sites during yeast meiosis. EMBO J. 13: 5754-5763.

Pan, J., M. Sasaki, R. Kniewel, H. Murakami, H. G. Blitzblau et al., 2011 A hierarchical combination of factors shapes the genome-wide topography of yeast meiotic recombination initiation. Cell 144: 719-731.

Panizza, S., M. A. Mendoza, M. Berlinger, L. Huang, A. Nicolas et al., 2011 Spo11-accessory proteins link double-strand break sites to the chromosome axis in early meiotic recombination. Cell 146: 372-383.

Penedos, A., A. L. Johnson, E. Strong, A. S. Goldman, J. A. Carballo et al., 2015 Essential and checkpoint functions of budding yeast ATM and ATR during meiotic prophase are facilitated by differential phosphorylation of a meiotic adaptor protein, Hop1. PLoS One 10: e0134297.

Perez-Hidalgo, L., S. Moreno, and P. A. San-Segundo, 2003 Regulation of meiotic progression by the meiosis-specific checkpoint kinase Mek1 in fission yeast. J. Cell Sci. 116: 259-271.

Preuss, U., G. Landsberg, and K. H. Scheidtmann, 2003 Novel mitosis-specific phosphorylation of histone $\mathrm{H} 3$ at Thr11 mediated by Dlk/ZIP kinase. Nucleic Acids Res. 31: 878-885.

Prugar, E., C. Burnett, X. Chen, and N. M. Hollingsworth, 2017 Coordination of double strand break repair and meiotic progression in yeast by a Mek1-Ndt80 negative feedback loop. Genetics 206: 497-512.

R Development Core Team, 2012 R: A Language and Environment for Statistical Computing. R Foundation for Statistical Computing, Vienna, Austria.

Rockmill, B., and G. S. Roeder, 1991 A meiosis-specific protein kinase homolog required for chromosome synapsis and recombination. Genes Dev. 5: 2392-2404.

Shimada, M., H. Niida, D. H. Zineldeen, H. Tagami, M. Tanaka et al., 2008 Chk1 is a histone $\mathrm{H} 3$ threonine 11 kinase that regulates DNA damage-induced transcriptional repression. Cell 132: 221-232.

Shinohara, A., H. Ogawa, and T. Ogawa, 1992 Rad51 protein involved in repair and recombination in $S$. cerevisiae is a RecAlike protein. Cell 69: 457-470.

Shroff, R., A. Arbel-Eden, D. Pilch, G. Ira, W. M. Bonner et al., 2004 Distribution and dynamics of chromatin modification induced by a defined DNA double-strand break. Curr. Biol. 14: 1703-1711.

Sikorski, R. S., and P. Hieter, 1989 A system of shuttle vectors and yeast host strains designed for efficient manipulation of DNA in Saccharomyces cerevisiae. Genetics 122: 19-27.

Smith, A. V., and G. S. Roeder, 1997 The yeast Red1 protein localizes to the cores of meiotic chromosomes. J. Cell Biol. 136: 957-967.

Sollier, J., W. Lin, C. Soustelle, K. Suhre, A. Nicolas et al., 2004 Set1 is required for meiotic S-phase onset, double-strand break formation and middle gene expression. EMBO J. 23: 1957-1967.

Sommermeyer, V., C. Beneut, E. Chaplais, M. E. Serrentino, and V. Borde, 2013 Spp1, a member of the Set1 Complex, promotes meiotic DSB formation in promoters by tethering histone H3K4 methylation sites to chromosome axes. Mol. Cell 49: 43-54.

Subramanian, V. V., and A. Hochwagen, 2014 The meiotic checkpoint network: step-by-step through meiotic prophase. Cold Spring Harb. Perspect. Biol. 6: a016675. 
Subramanian, V. V., A. J. MacQueen, G. Vader, M. Shinohara, A. Sanchez et al., 2016 Chromosome synapsis alleviates Mek1dependent suppression of meiotic DNA repair. PLoS Biol. 14: e1002369.

Suhandynata, R. T., L. Wan, H. Zhou, and N. M. Hollingsworth, 2016 Identification of putative Mek1 substrates during meiosis in Saccharomyces cerevisiae using quantitative phosphoproteomics. PLoS One 11: e0155931.

Sun, X., L. Huang, T. E. Markowitz, H. G. Blitzblau, D. Chen et al., 2015 Transcription dynamically patterns the meiotic chromosomeaxis interface. Elife 4: e07424.

Thacker, D., N. Mohibullah, X. Zhu, and S. Keeney, 2014 Homologue engagement controls meiotic DNA break number and distribution. Nature 510: 241-246.

Tung, K. S., E. J. Hong, and G. S. Roeder, 2000 The pachytene checkpoint prevents accumulation and phosphorylation of the meiosis-specific transcription factor Ndt80. Proc. Natl. Acad. Sci. USA 97: 12187-12192.

Usui, T., H. Ogawa, and J. H. Petrini, 2001 A DNA damage response pathway controlled by Tel1 and the Mre11 complex. Mol. Cell 7: 1255-1266.

Wal, M., and B. F. Pugh, 2012 Genome-wide mapping of nucleosome positions in yeast using high-resolution MNase ChIP-Seq. Methods Enzymol. 513: 233-250.

Wan, L., T. de los Santos, C. Zhang, K. Shokat, and N. M. Hollingsworth, 2004 Mek1 kinase activity functions downstream of RED1 in the regulation of meiotic double strand break repair in budding yeast. Mol. Biol. Cell 15: 11-23.
Weiner, A., A. Hughes, M. Yassour, O. J. Rando, and N. Friedman, 2010 High-resolution nucleosome mapping reveals transcription-dependent promoter packaging. Genome Res. 20: 90-100.

Wu, T.-C., and M. Lichten, 1994 Meiosis-induced double-strand break sites determined by yeast chromatin structure. Science 263: 515-518.

Xu, L., M. Ajimura, R. Padmore, C. Klein, and N. Kleckner, 1995 NDT80, a meiosis-specific gene required for exit from pachytene in Saccharomyces cerevisiae. Mol. Cell. Biol. 15: 6572-6581.

Xu, L., B. M. Weiner, and N. Kleckner, 1997 Meiotic cells monitor the status of the interhomolog recombination complex. Genes Dev. 11: 106-118.

Yang, W., Y. Xia, D. Hawke, X. Li, J. Liang et al., 2012 PKM2 phosphorylates histone $\mathrm{H} 3$ and promotes gene transcription and tumorigenesis. Cell 150: 685-696.

Zakharyevich, K., Y. Ma, S. Tang, P. Y. Hwang, S. Boiteux et al., 2010 Temporally and biochemically distinct activities of Exo1 during meiosis: double-strand break resection and resolution of double Holliday junctions. Mol. Cell 40: 1001-1015.

Zickler, D., and N. Kleckner, 1999 Meiotic chromosomes: integrating structure and function. Annu. Rev. Genet. 33: 603-754.

Zickler, D., and N. Kleckner, 2015 Recombination, pairing, and synapsis of homologs during meiosis. Cold Spring Harb. Perspect. Biol. 7: a016626. 